\title{
Talking at Cross-Purposes: How Einstein and the Logical Empiricists never Agreed on what they were Disagreeing about
}

\author{
Marco Giovanelli
}

\begin{abstract}
By inserting the dialogue between Einstein, Schlick and Reichenbach into a wider network of debates about the epistemology of geometry, this paper shows that not only did Einstein and Logical Empiricists come to disagree about the role, principled or provisional, played by rods and clocks in General Relativity, but also that in their lifelong interchange, they never clearly identified the problem they were discussing. Einstein's reflections on geometry can be understood only in the context of his "measuring rod objection" against Weyl. On the contrary, Logical Empiricists, though carefully analyzing the Einstein-Weyl debate, tried to interpret Einstein's epistemology of geometry as a continuation of the Helmholtz-Poincaré debate by other means. The origin of the misunderstanding, it is argued, should be found in the failed appreciation of the difference between a "Helmholtzian" and a "Riemannian" tradition. The epistemological problems raised by General Relativity are extraneous to the first tradition and can only be understood in the context of the latter, the philosophical significance of which, however, still needs to be fully explored.
\end{abstract}

Keywords: Logical Empiricism Moritz Schlick, Hans Reichenbach, Albert Einstein, Hermann Weyl, Epistemology of Geometry

\section{Introduction}

Mara Beller in her classical Quantum dialogue (Beller, 1999) famously suggested a "dialogical" approach to the history of science. According to Beller, the scientific discourse always addresses the discourse of other scientists, who in turn may react by changing their views in response. In Beller's view, scientific discourse is not only dialogical but also "polyphonic". Different dialogues overlap and nurture each other to form a communicative network, the analysis of which is indispensable in understanding the single contributions.

Beller of course provided her own celebrated dialogical analysis of the emergence of the so-called "Copenhagen interpretation" of quantum mechanics. Such a methodological approach, however, could be effectively extended to the history of philosophy of science. In particular there is little doubt that the dialogue about the epistemology of geometry between Albert Einstein and the Logical Empiricists, mainly Moritz Schlick and Hans Reichenbach, represents one of the decisive moments, if not the decisive moment, of 20th century philosophy of science (Howard, 2009). In this dialogue Logical Empiricists established the foundation of their new kind of empiricism which, for better or worse, inspired a generation of philosophers.

As we shall see, this dialogue is related to other dialogues: the debate between Einstein, Max Born, Paul Ehrenfest, Max von Laue and others around the 1910s on

Email address: marco.giovanelli@uni-tuebingen.de (Marco Giovanelli) the notion of rigid bodies in Special Relativity (\$2.1), and most of all to the debate between Einstein, Herman Weyl and Walter Dellänbach (§3) on the role of rigid rods and clocks in General Relativity (\$2.2) around the 1920s. In turn, these dialogues bear a complex relation to a series of controversies, between Bernhard Riemann, Herman von Helmholtz, Henri Poincaré and others, which burst forth in the second half on 19th century after the "discovery" of non-Euclidean geometries (Torretti, 1978).

Einstein's dialogue with Schlick and Reichenbach must then be understood against a backdrop of this rich and variegated web of interrelated dialogical interchanges, in which every argument reveals its full meaning only when regarded as a response to other arguments. The aim of this paper is to show that this dialogue, considered in its proper historical setting, can be singled out by a sort of peculiarity: its protagonists never really agreed on what they where discussing and, surprisingly, never seemed to have noticed.

In recent literature the Einstein-Logical Empiricists debate has been regarded as a philosophical controversy concerning a problem that the "parties to the instrument" univocally identified: whether measuring rods and clocks do or should play an epistemologically fundamental role in General Relativity. Einstein, until the end of his life, argued that the theory has empirical content, as soon as one assumes the existence of rigid infinitesimal rods that can be used to measure the interval between any two neighboring points (Stachel, 1989). However, he also recognized on several occasions that this was only a sort of practical com- 
promise (Howard, 1990, 1994), or at least he was forced by Weyl to recognize it (Ryckman, 1995). Both Weyl and the Logical Empiricists can then be considered as "Einstein's agonists", even if they were speaking up for "different Einsteins" (Ryckman, 1996, 2005), or perhaps two different sides of the "same Einstein" (Howard, 2005, 2009).

The "received view" can then be roughly summarized as follows: In the 1920s the Logical Empiricists, especially Schlick and Reichenbach, were convinced they were following Einstein's guidance in assuming that without rods and clocks the "edifice of physics" would be deprived of empirical content (Friedman, 2002); however, Einstein, more in consonance with Weyl, accepted this assumption only provisionally: the behavior of rods and clocks should be in principle derived from the theory and not used to grant its observational basis (Ryckman, 2005).

Recent historical literature had thus made enormous progress in revealing the complex "dialogical" background hidden behind the apparently monolithic Einstein-Logical Empiricists-epistemology of geometry (see Fogel, 2008, ch. 3, 4 for an excellent overview). Einstein's position turned out to be much more sophisticated than the Logical Empiricists had believed, and most of all rather at odds with their own empiricism. In my opinion, however, recent literature has been strangely recalcitrant in making the successive step to unraveling a rather discomforting truth about the "emperor's clothes". Actually Einstein and the Logical Empiricists were simply not discussing the same problem. The celebrated dialogue to which modern philosophy of science owes its origin was a dialogue of the deaf.

Logical Empiricists were convinced that the HelmholtzPoincaré controversy over the empirical-conventional choice among the class of possible (Euclidean or non-Euclidean) Riemannian geometries could be extended in the new general relativistic context (Friedman, 1995), by substituting a stipulation about the congruence of "finite rigid bodies" with that of "infinitesimal rigid rods" (Torretti, 1983, 239f.). Einstein was rather exclusively concerned with the very existence of rigid infinitesimal rods, which is presupposed by Riemannian geometry, but - as Weyl had shown - is far from being necessary. Einstein's rather sporadic references to the Helmholtz-Poincaré debate served then to meet Weyl's challenge rhetorically in semi-popular writing, rather then make a case for conventionalism $(\S 4.2 ; \S 4.5)$.

As we shall see in detail, the Logical Empiricists knew the issue raised by Weyl very well and discussed it at length — in particular Reichenbach — along with Einstein's counter-objection, thereby displaying a vast knowledge of all technical details $(\S 4.1 ; \S 4.3 ; \S 4.4 ; \S 5)$; however, they surprisingly never came to realize that Einstein's epistemological reflections on geometry precisely addressed Weyl's criticism of the use of rods and clocks as direct metrical indicators. Even more puzzling is the fact that Einstein, in turn, apparently never felt compelled to clear up the misunderstanding, neither in private correspondence nor in published writings, nurturing the reciprocal illusion that the debate revolved around a well- identified problem to which the contenders gave different solutions.

The historical dialogue between Einstein and the Logical Empiricists found its crowning moment in the famous imaginary dialogue between "Reichenbach" and "Poincaré" staged by Einstein in his final "Remarks" for the Library of Living Philosopher's volume published in the late 1940s. As Reichenbach's successive reply shows, even on that occasion, Einstein and Reichenbach did not come to an agreement on what the apple of discord was (§6). For more than thirty years Einstein, Schlick and Reichenbach were engaged in a lively discussion about the epistemology of geometry, but for more than thirty years they were talking at cross-purposes.

If our reconstruction turns out to be correct, we then have to face the ugly truth that the epoch-making debate in which philosophy of science was defined as an independent discipline was based in hindsight on a quite simple misunderstanding. There are still, however, some relevant lessons that can be drawn from an accurate historical reconstruction of this debate. In contrast to what the Logical Empiricists thought and what it is still often argued in the literature, what we may call the "Helmholtzian" tradition which traversed 19th century geometry did not play any relevant role in the emergence of General Relativity, and is utterly inadequate to understand the philosophical problems that theory raised.Einstein's theory drew its conceptual resources exclusively from what we may call a "Riemannian tradition", that, although evolving in parallel during roughly the same years, philosophers have often neglected to adequately investigate $(\S 7)$.

\section{From Finite Rigid Bodies to Infinitesimal Rigid Rods: Einstein between Geometry and Reality}

\subsection{Finite Rigid Bodies in Special Relativity}

Early on, Einstein insisted on the "epistemological" importance of "rigid bodies" as the mediating element to connect geometry with physical reality. Already in his 1905 paper on the electrodynamics of moving bodies, Einstein pointed out that in a coordinate system at rest the position of a body can be determined "by the employment of rigid measuring rods [Maßstäbe] under the methods of Euclidean geometry and expressed in Cartesian co-ordinates" (Einstein, 1905, 892). A Cartesian system of co-ordinates can be thought of as a cubical framework formed by rigid unit rods. Translations of such a rigid cube into itself can be used to introduce number triples as coordinates and these can then be employed as marks of position throughout the entire space. To specify relations in time, we require in addition a standard clock (in general a system which runs down periodically) placed, say, at the origin of our Cartesian system of coordinates or frame of reference. In this context, the introduction of coordinates could therefore be directly defined by measurements made by rigid bodies and uniformly ticking clocks (see for instance Einstein, 1907, 439). 
As early as 1907, however, Einstein pointed out that in Special Relativity it is difficult to clarify the behavior of bodies and clocks in accelerated frames of reference (Einstein, 1907, §18; in particular 455). In 1909 Max Born (Born, 1909), referring to Einstein's 1907 paper, suggested a Lorentz invariant definition of a rigid body (the so called Born rigidity condition). "The method used by me," Born writes, "consists in defining rigidity by a differential law instead of an integral law" (Born, 1909, 3); in particular Born defined analytically "the differential conditions of rigidity" by using a quadratic form of three spacial differentials (Born, 1909, §2 ).

In addition to the paper published in the Annalen der Physik, Born presented the results of his work at the meeting of the German Society of Scientists and Physicians (Salzburg, 21-25 September 1909), which was also attended by Einstein. The results were published one year later (Born, 1910). On that occasion Born mentioned Gustav Herglotz's result that in Special Relativity a rigid body has only three degrees of freedom, in contrast to the six of classical mechanics (Herglotz, 1910; see also Noether, 1910). Just four day after the meeting Paul Ehrenfest published in Physikalische Zeitschrift a celebrated paper discussing how an ideally rigid cylinder cannot be brought from rest into a state of rotation about its axis of symmetry, without violating Lorentz Invariance (Ehrenfest, 1909). The paradox became well known as the "Ehrenfest Paradox" (cf. Grøn, 2004).

In private correspondence Einstein declared his interest in "[t]he latest relativity-theoretical investigations of Born and Herglotz ... It really seems," he argued, "that in the theory of relativity there does not exist a 'rigid' body with 6 degrees of freedom" (to in Jakob Laub, March 1910, CPAE: 5. 232; tr. in Stachel, 1989). In 1911 Max von Laue, developing the works of Born, Ehrenfest, Herglotz and Noether, showed that in Special Relativity an accelerated body has an infinite amount of degrees of freedom, thus implying that rigid bodies cannot exist. By assuming that "a propagation with superluminal speed is excluded for all physical effects" (von Laue, 1911, 86), Laue showed that an impulse simultaneously acting on $n$ different points of a body will necessarily result in at least $n$ degrees of freedom. Einstein explicitly mentioned a similar result in a discussion following the lecture version of Die RelativitätsTheorie (Zurich, 16 January 1911; published as Einstein, 1911): "There can be altogether no rigid body according to the Theory of Relativity" (CPAE 3, Doc. 17, 443). If we move a part of a rigid body then also the other end should immediately move. However, this would be an infinitely fast signal which is not acceptable in Special Relativity.

Thus, although Einstein did not participate publicly in the discussion of the Ehrenfest Paradox, he was well aware of the difficulties that lurk in the definition of a "rigid body" in Special Relativity (cf. Sauer, 2008). However, the concept of "rigid body" apparently continued to play for him the fundamental "epistemological" role of mediating between abstract geometry and physical geometry: "the propositions of Euclidean geometry," Einstein noticed in his 1912 lectures on Special Relativity "obtain physical content", if they are interpreted as "propositions concerning arrangements [Gruppierungen] of material straight lines and tracts in relative rest" (CPAE 4, Doc. 1, 36).

In a footnote attached to this passage, Einstein labeled this definition as "woolly [unscharf] (Rotation)" (CPAE 4, Doc. 1,$104 ;$ n. 56). The reference is probably to the the famous "rigidly rotating disk" thought experiment (Stachel, 1989), which Einstein mentioned for the first time in a published paper in February 1912 (Einstein, 1912, §1). Einstein came to the conclusion - reached independently by Theodere Kaluza (Kaluza, 1910) — that the propositions of Euclidean geometry cannot hold exactly on the rotating disc nor in general within a gravitational field. However, according to Einstein, "[t]he measuring rods as well as the coordinate axes are to be considered as rigid bodies", "even though the rigid body cannot possess real existence" (Einstein, 1912, 131).

\subsection{Infinitesimal Rigid Rods and General Relativity}

As Abrahm Pais has noted, "the celebrated problem of the rigid body in the special theory of relativity stimulated Einstein's step to curved space, later in 1912" (Pais, 1982, 202). Einstein was forced to rethink the relationship between coordinates and measurements with rods and clocks (Stachel, 1989) and to abandon the restrictions imposed by Euclidean geometry; moreover Born's mathematical technique may have led him to consider quadratic differential forms with variable coefficients (Maltese and Orlando, 1995). As is well known, around 1912 Einstein was introduced to the work of Erwin Bruno Christoffel (Christoffel, 1869), Gregorio Ricci-Curbastro and Levi-Civita (LeviCivita and Ricci-Curbastro, 1900) by his mathematician friend Marcel Grossmann. In 1913, together with Grossmann, Einstein saw how to formulate, at least in outline, a theory of gravitation based on the expression $d s^{2}=$ $\sum g_{\mu \nu} d x_{\mu} d x_{\nu}$, whose coefficients $g_{\mu \nu}$ represent the behavior of measuring rods and clocks with reference to the coordinate system, as well as the potentials of the gravitational field (Einstein and Grossmann, 1913).

In this context, co-ordinates lost their simple metrical significance and do not directly signify lengths measured by a unit measuring rod any more: The length of a measuring rod is not determined solely by the coordinate differentials $d x_{1}, d x_{2}, d x_{3}$ but also by the six functions $g_{11}, g_{22}, g_{33}$. In a four dimensional manifold the measure of time is similarly influenced by the gravitational potential $g_{44}$, so that the distance of two neighboring events, measured with the aid of a portable clock, is different from the differential $d x_{4}$ of the time coordinate: "From this one sees that, for given $d x_{1}, d x_{2}, d x_{3}, d x_{4}$ " the interval $d s$ which "corresponds to these differentials can be determined only if one knows the quantities $g_{\mu \nu}$ that determine the gravitational field" (Einstein and Grossmann, 1913, 9). Thus, one does not know the meaning of "distance" 
between two events, specified initially by physically meaningless co-ordinates $x_{\nu}$, until one knows the coefficients $g_{\mu \nu}$. On the other hand, after a coordinate system has been introduced, the $g_{\mu \nu}$ should in principle be obtained from the direct measurement of space-like and time-like distances.

In the Entwurf-paper, Einstein found the solution to this conundrum by assuming that one can construct a Euclidean or Minkowskian coordinate-system with unit rods and clocks in an small enough (in astronomical proportions) region of space-time. Instead of the general coordinates $x_{\nu}$, one can introduce the rectangular coordinates $\xi_{\nu}$, in which $d s^{2}=d \xi_{1}^{2}+d \xi_{2}^{2}+d \xi_{3}^{2}+d \xi_{4}^{2}$, i.e. Special Relativity is valid, where the $g_{\mu \nu}$ are constant; the invariant measure of the distance between two space-time points $d s$, as determined by unit rods and clocks not accelerated in this frame, is referred to by Einstein as "naturally measured" (Einstein and Grossmann, 1913, 8).

In the last sections of the successive systematic exposition of the Entwurf-theory, Die formale Grundlage der allgemeinen Relativitätstheorie (Einstein, 1914) — presented to the Berlin Academy in 1914 - Einstein suggested the implications that the new theory could have on the philosophy of geometry. Traditionally geometry presupposes that 'two points of a 'rigid' body must be separated by a certain distance, which is independent of the position of the bodies; the propositions of geometry completed with this statement are (in a physical sense) either right or wrong" (Einstein, 1914, 1079; my emphasis). These propositions are considered as integral laws, since they "deal with distance of points at a finite region" (Einstein, 1914, 1079). General Relativity has forced us to adopt a near-geometrical approach - akin to that of Maxwell in physics - by "gradually introducing the requirement that the finite distances between points could no longer appear in the elementary laws" (Einstein, 1914, 1080; my emphasis). Rigid finite bodies are substituted by infinitesimal rigid rods so that the propositions of geometry "are reduced to differential laws" (Einstein, 1914, 1080; cf. D'Agostino, 2000, 235).

After having presented the field equations for determining the $g_{\mu \nu}$ in November 1915 (Einstein, 1915c,d,a,b), in $\S 4$ of his March 1916 review paper Die Grundlage der allgemeinen Relativitätstheorie (Einstein, 1916), Einstein returned to the conditions necessary to compare the predictions of the theory with the observed facts. He insisted again on the fact that " $d s^{2}$ is a quantity ascertainable by rod-clock measurement of point-events infinitely proximate in space-time, and defined independently of any particular choice of co-ordinates" (Einstein, 1916, 776). More precisely the $g_{\mu \nu}$ can be found stepwise in the failure to extend the local Minkowski coordinates over larger regions of space-times; they are the numbers to which we have to multiply the coordinate distances in order to assure $d s^{2}$ has the same length all over the manifold. Of course the same length can be determined only up to an arbitrary constant; so one still needs to make a global choice of the "units in which we shall express the length of lines" (Lorentz, 1917,
$1345)$.

\subsection{Atoms and Clocks}

To appreciate the physical meaning of this last remark, it is useful to look at a paper that the Austrian Physicist Ludwig Flamm sent to the Physikalische Zeitschrift in September 1916. Illustrating the Schwarzschild solution (Schwarzschild, 1916) in a geometrical form, Flamm explains with admirable clarity under which conditions it is possible to perform measurements in General Relativity:

If one chooses as an elementary clock the red line emitted by a cadmium atom [die rote Kadmiumlinie aussendende Molekül] and sets its period of oscillation as the time unit, then one recognizes at once [ohne weiteres] that, because of the constancy of the velocity of light [wegen jener Konstanz der Lichtgeschwindigkeit], the metrical unit length at every place and in every time must coincide [decken muss] with the same number of wave lengths of the cadmium red line [der roten Kadmiumlinie]. Similarly, if one set an elementary measuring-rod as the lattice spacing [Distanzgitter] of the rock-salt crystal [Steinsalzkristalls], one would reach the same conclusion in relation to the "naturally measured" velocity of light. This fundamental presupposition lies at the basis of General Theory of Relativity, that for instance the ratio between the wave-length of the red cadmium line and the lattice constant of rock salt is an absolute constant. Also in an arbitrary gravitational field, this constant must be from independent of place and orientation and of the time point [Zeitpunkt] (Flamm, 1916, 451; my emphasis).

General Relativity is built on the assumption that ideal clocks are not affected by the presence of a gravitational field. If two cadmium atoms are brought together to the same place, they will always show the same frequency, $6436.4696 \AA$, for the cadmium red line (Jackson, 1936). If we set this rate of ticking as the unit clock, we have then defined a time unit which can easily be reproduced all over the manifold, since all cadmium atoms' red lines will show the same frequency and same rate of ticking. Of course, the particular standard clock used does not matter because the only effect of changing from one clock to another is to change the unit of time with the ratio of the two units being constant. As Flamm points out, the ratio of the wavelength of the red cadmium spectral line over the lattice distance of rock-salt crystal is an absolute constant of nature.

Referring to Lamm's paper, among others, in his lectures on the foundations of physics held in Winter Term 1916-1917, David Hilbert seems to already be clear about the epistemological difficulties which lurk behind this assumption:

In order to read the time one needs a clock. Such a clock can be found realized in nature in the greatest conceivable perfection in an oscillating and lightemitting atom [Molekül], let's say a yellow spectral 
line of the sodium atom ... The proper time $T$ of an oscillating atom is independent of the gravitational field, in which the atom is located ... This axiom has of course ... only a provisional character. When physics will be finally fully developed [vollständig ausgebaut], then the axiom must appear as a consequence of the general theory (Hilbert, 1917, 284; my emphasis)

In order to perform measurements in General Relativity one must assume as an axiom that, for instance, the yellow emission line of sodium vapor measured by an observer at rest with respect to the atom will always be $5893 \AA$. If we set this as the unit of length, the ticking of such clocks provides a measure of the length of a time-like world-line. Two identical clocks in different positions within a gravitational field ( let's say, on the earth and on the sun) measure, of course, a different elapsed time (first clock effect or twin paradox). As a consequence, the frequency of a signal emitted by one clock would appear to be increased (red shift) or decreased (blue shift) if measured at the position of the other clock (Hilbert, 1917, 285; cf. Earman and Glymour, 1980; Hentschel, 1994). However, the rate of ticking of identical clocks, as measured by a nearby observer, is assumed to be independent of the world-line on which the measurements are made. Such an "axiom" allows the comparison of lengths measured at a distance as Riemannian geometry requires.

At the end of the passage just quoted, Hilbert makes an epistemological point that cannot fade into silence. General Relativity assumes this "axiom", but it cannot prove it through its own conceptual resources. Thus this assumption has only a provisional character. At a later stage of development of physics it should appear, however, as the consequence of the theory. Even if it is hard to track down Hilbert's sources, his phrasing reveals a clear similarity with that of Einstein, who, as we shall see, had started at about the same time to attribute to this very issue a fundamental epistemological importance, which contemporary philosophers, alas, have not always been able to appreciate.

\section{Schlick's Early Conventionalism and Einstein's Discussion with Weyl and Dällenbach}

As is well known, Einstein's insistence on the importance of measurement rods and clocks was the starting point of Moritz Schlick's attempt to provide a philosophical interpretation of General Relativity, exemplified in his celebrated 1917 paper, published in two installments on the semi-popular journal Die Naturwissenschaften (Schlick, 1917, tr. in Schlick, 1978, vol. I, 207-269). From Schlick's point of view Einstein's approach could be considered the heir to the discussion between Helmholtz and Poincaré on the role of rigid bodies in the epistemology of geometry. In particular, Schlick introduced here the main lines of his conventionalism: the choice of the geometry of space depends on which body one considers as rigid; but it is impossible to know which bodies are rigid without knowing the geometry of space. One breaks the circle by regarding as rigid those bodies from which the simplest physics would result, that is, the physics that contain the least arbitrary elements (Schlick, 1917, 167).

Of course Schlick was well aware that "the notion of a rigid rod, which preserved the same length at all times, no matter what its position and surroundings might be" (Schlick, 1917, 182, tr. 1978, I, 238), "had already been modified to a certain extent in the Special Theory of Relativity", where "the condition was fulfilled", only for "a rod respectively at rest with regard to each system in question" (Schlick, 1917, 182, tr. 1978, I, 239). In General Relativity "the length of a rod ... can also depend on its place and its position", so that the very possibility of measurements was irremediably jeopardized (Schlick, 1917, 183, tr. 1978, I, 244).

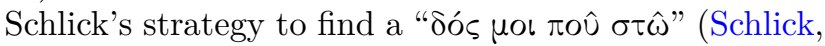
1917, 183, tr. 1978, I, 244) to perform measurements within a general relativistic context was of course to assume, as Einstein did, that the behavior of rigid rods is Euclidean (or Minkowskian in case of rods and clock) in small domains - which, however, "may still be large compared with the dimensions which are used elsewhere in physics" (Schlick, 1917, 183, tr. 1978, I, 245). In small portions of space-time, as Schlick points out, "[t]he 'line-element' has a direct physical meaning, and can be ascertained by means of measuring-scales and clocks" (Schlick, 1917, 183, tr. 1978, I, 245), independently of the coordinate system used. It must then be assumed that the "the value which we there obtain for $d s$ is valid generally" (Schlick, 1917, 184 , tr. 1978, I, 249), i.e. the line element $d s$ has the same length under all circumstances: "The numerical value of $d s$ is always the same, whatever orientation the chosen local co-ordinate system may have" (Schlick, 1917, 183, tr. 1978, I, 245).

Schlick offers an accurate reconstruction of Einstein's own theory of measurement; its compatibility with Schlick's own conventionalism is, however, all but obvious. On Schlick's own account, the rigidity of small rods is not stipulated: rigid are those rods and ideal are those clocks that have a pseudo-Euclidean behavior; meaning those that can be disposed to forming a rectangular grid in sufficiently small regions of space-time (Torretti, 1983, 239f.). One of these measuring devices can be set conventionally as a unit rod, but this has nothing to do with the conventional choice among different possible metrical geometries. Changing the unit of measurement does not change the metric of space but only multiplies all lengths by the same scale factor. In Riemannian geometry, this factor is in fact assumed to be constant, i.e. that ratio of the two units is assumed to be the same whenever they are compared.

Schlick, who had been in correspondence with Einstein since the late 1915, sent him a copy of his paper on the 4th of February, 1917. Einstein replied on the 6th of February, 1917 praising Schlick's paper unconditionally, espe- 
cially for its empiricist flavor (Howard, 1984; Hentschel, 1986). However, some days later (probably after the 15 th of February, 1917), Einstein wrote to his former Zurich student Walter Dällenbach, stressing that, from an epistemological point of view, the idea that the $d s$ could be found directly through a measurement done by rigid rods is not at all satisfying :

Dear Dällenbach! Your remarks are, in my opinion, to a large extent correct. ${ }^{1}$ Strictly speaking, the concept of $d s^{2}$ volatilizes in an empty abstraction: $d s^{2}$ cannot be rigorously considered as the result of measurements, not even in the absence of electromagnetic fields. You have rightly indicated the reasons why it is so. Nevertheless, in a didactically reasonable presentation of the theory the $d s^{2}$ must be so considered, as if it were rigorously measurable ... A logically satisfying presentation can be achieved (a posteriori), so that the a single, more complex solution is related to the observed facts. A measuring-rod would then be an atomic system of a certain type that does not play any special role in the theory (CPAE 8, Doc. 565, 803; my emphasis)

A small rigid rod in astronomical proportions, let's say a rock-salt crystal, is a composite structure in atomically; its rigidity results from the fixity of the spacing between the chlorine and sodium atoms arranged in a cubic lattice, maintained by electrical forces. Thus, rigid rods cannot be considered as theoretically self-sufficient entities, but their description involves quantum theoretical principles, whose justification, however, lies outside the conceptual framework of General Relativity. As Einstein admits, the behavior of such an atomic structure, the fact that it preserves its length wherever it is transported, should in principle be justified within General Relativity, rather than serving to connect it with the observed facts.

As the letter to Dällenbach reveals, Einstein's repeated insistence on measuring rods and clocks, far from representing the chief philosophical implications of the General Relativity, as Schlick thought, was then a sort of provisional and "didactic" compromise, a point on which, as we have seen, Hilbert had also insisted nearly at the same time. Dellänbach was at that time Weyl's doctoral student; his objection to Einstein probably reflects the discussions that he might have had with Weyl himself. In 1917, Weyl gave his famous lectures on relativity at the ETH Zurich, which would appear as a book one year later in 1918 with the title Raum-Zeit-Materie.

As is well known, in his presentation Weyl explicitly tried to avoid the use of exceedingly complicated measuring instruments, such as rods and clocks, for the measurement of $g_{i k}$ by restricting himself exclusively to the observation of the arrival of light signals; for instance, those emitted by two stars, i.e. to measuring the angle between

${ }^{1}$ Einstein refers to a private conversation. null geodesics $\left(d s^{2}=0\right)$ at a certain point. In this way, however, the metric can be determined only up to an arbitrary scale factor $\left(g_{i k}^{\prime}=\lambda g_{i k}\right)$, which "can be determined only through the individual choice of a unit of measure" (Weyl, 1918c, 182). After determining the metric up to a constant by the use of light rays, one can use rods and clocks to determine the constant as well, by fixing a choice of units for space-time distances. The constancy of $\lambda$ assures the comparability at a distance not only of the angles among curves, but also of their lengths.

In a 1918 paper, Reine Infinitesimalgeometrie, Weyl famously argued, however, against the necessity of this last element of "geometry at a distance" (Weyl, 918s) which still survives in Riemannian Geometry (form more details cf.: Scholz, 2008, 2004; Ryckman, 2005; Bell and Korté, 2011). All intervals between close pairs of points can thus be changed by the multiplication of an arbitrary factor $\lambda$, which may be different in different locations. As is well known, in a successive paper, Gravitation und Elektrizität (Weyl, 1918b), Weyl suggested that, by removing such a blemish in Riemannian geometry, in addition to the $g_{\mu \nu}$ also the quantities $\phi_{\nu}$ appear, which could be identified with the four potentials of the electromagnetic field (Weyl, 1918b).

As we have seen, in Einstein's theory it is presupposed that if two cadmium atom clocks are separated and brought together along different world-lines, they will still tick at equal rates, but in general will show a "first or usual clock effect"; from this they would then measure different elapsed times, if one of them had passed through a gravitational field. Weyl's theory would introduce a "second clock effect": If two cadmium atoms are separated and brought back, the final size of the atoms might differ, the spectral lines might be shifted, and thus would show different rates of ticking that would serve as an indicator of the electromagnetic field (cf.: Vizgin, 1994; O'Raifeartaigh and Straumann, 2000; Goenner, 2004, ch. 3; § 4.1).

Einstein was highly impressed by Weyl's theory, but he was unable to settle "the measuring-rod objection [MaßstabEinwand]" against it, which he communicated personally to Weyl in March 1918 (CPAE 8, Doc. 510 and 512). In a brief note published in an appendices to Weyl's paper (Einstein, 1918), Einstein famously argued that if Weyl's theory were true, "there could not be chemical elements with spectral lines of determined frequencies"; rather, "the relative frequencies of two spatially neighboring atoms of the same kind should be, in general, different" (Einstein, $1918,40)$. However, this is not the case. The relative constancy of the frequency of atoms is then a plausible factual assumption. As Weyl pointed out in his rejoinder to Einstein, however, "the task remains, in my theory as well as in Einstein's, to derive this fact by the dynamics carried through explicitly" (Weyl, 1918a, 479).

Writing to Einstein (after the 15th of June, 1918), Dellänbach acknowledged that, if Weyl's theory applied to reality, "one would lose the connection with the observed facts" (CPAE 8, Doc. 565, 803); however, in order to as- 
sure this connection, Einstein had assumed "too complex things as rigid bodies or clocks" (CPAE 8, Doc. 565, 803) while being unable to explain their behavior. Einstein's reply is interesting throughout:

\begin{abstract}
If then two $d s$ at distant points were measured with measuring rods or, in a certain way, were found to be equal, they would still be found equal, if they were measured in a different way. This is a deep property of our world, which must find expression in the foundation of physics... I know that Weyl does not admit it. He would say that clocks and measuring-rods must appear as solutions; they do not occur in the foundation of the theory. But I find: if the $d s$ is measured by a clock (or a measuring-rod), is something independent of prehistory, construction and the material, then this invariant as such must also play a fundamental role in the theory. Yet, if the manner in which nature really behaves would be otherwise, then spectral lines and well-defined chemical elements would not exist (CPAE 8, Doc. 565, 803; my emphasis)
\end{abstract}

In other words, Einstein could only acknowledge a res facti, that we happen to live in a world in which the relative periods of clocks and the relative lengths of rods do not depend on their histories. Weyl, on the contrary, was raising a questio iuris by asking that the behaviors of such complicated material structures, such as rods and clocks, should be considered as a solution to the equations of the theory; (in Einstein's as well as in his own theory), that is, it should be deduced from some dynamical theory of matter (cf. Weyl, 1919, 260).

Following this line of thought by 1920 (Weyl, 1920), at the Meeting of Natural Scientists in Bad Nauheim (1925 September 1920), Weyl had started to account for the apparent constancy of frequency of atoms by distinguishing between a "tendency of persistence" (Beharrungstendenz) or "adjustment" (Einstellung) (Weyl, 1920, 649). Roughly, Weyl suggested that cadmium atoms do not preserve their size if transported, but they adjust it every time to the radius of the spherical curvature of every threedimensional section of world (Weyl, 1920, 650). Similarly, a magnetic needle always adjusts its direction to the north in the earth's magnetic field and does not preserve it.

In the discussion which followed Weyl's paper, Einstein insisted, however, that in his theory "[t]emporal-spatial intervals are physically defined with the help of measuring rods and clocks" (Einstein reply to Weyl, 1920, 650), whose "equality is empirically independent of their prehistory". This presupposition — he argued — assures "the possibility of coordinating [zuzuordnen] a number, $d s$, to two neighboring points" (Einstein's reply to Weyl, 1920, 650). Renouncing this "coordination [Zuordnung]" would rob "the theory of its most solid empirical support and possibilities of confirmation" (Einstein's reply to Weyl, 1920, $650)$.

These and similar expressions were, of course, music to the Logical Empiricists' ears. However, Einstein, in the same Bad Nauheim meeting, by addressing other interlocutors, assumed a quite different stance. For instance, when discussing Max von Laue's paper on the gravitational redshift, Einstein defined, "the fact that measuringrods and clocks have to be introduced separately, instead of being constructed as solutions of differential equations" as "[a] logical weakness of the theory in the today state" (Einstein's reply to Laue, 1920, 652).

\section{The Emergence of Logical Empiricism as a Mis- understanding of Einstein's Philosophy of Ge- ometry}

\subsection{Reichenbach's Objections to Schlick's Conventional- ism}

Unaware of this complex dialogical background, in 1917 Schlick had, after all, very good reasons to emphasize the fundamental role that rods and clocks played in General Relativity. As Einstein wrote in a letter to Cassirer in June 1920: "The destiny of General Relativity as a physical theory depends entirely upon the interpretation of the $d s$ as result of measurement, which can be obtained in a quite definite way through measuring-rods and clocks" (CPAE 10, Doc. 44, 293). Schlick could then legitimately interpret Einstein's language of coordination as a confirmation of his own philosophical approach, which in the meantime had been presented in a systematic form in the first 1918 edition of the Allgemeine Erkenntnisslehre (Schlick, 1918), a book which, as we shall see, was to have a considerable influence on Einstein himself.

More puzzling is the fact that Schlick could believe that Einstein's insistence on the the direct measurability of the $d s$ was compatible with a form of conventionalism à la Poincaré. The impossibility of carrying out such a program is explained very neatly in Reichenbach's 1920 "Kantian" monograph on relativity, Relativitätstheorie und Erkenntnis apriori (Reichenbach, 1920)

As is well known, according to the young Reichenbach, who had been one of the five students in Einstein's first seminar on General Relativity, conventionalism works only for spaces of constant curvature (Friedman, 1995). In each of these spaces there is a unique (up to a constant positive factor - i.e. up to the choice of a "unit of length") set of congruence relations so that each such set of congruence relations is inconsistent with any other set. Conventionalism is then based precisely on the idea that one can make an arbitrary choice among one of these incompatible sets.

In Riemannian geometry of variable curvature, no unique set of congruence relations can be defined over all the space, so the very idea of a unique conventional choice among alternative congruent relations does not make sense. For this reason, Reichenbach points out, Poincaré "excludes from the beginning Riemannian geometry, because it does not permit the displacement of a body without change of form" (Reichenbach, 1920, 104, n. 1; tr. 1965, 109, 1; translation modified). In the general case only 
the unit of length is globally available on a Riemannian manifold, in contrast to Weyl's non-Riemannian geometry where a separate unit of length at every point of space may be defined.

What characterizes Einstein-Riemann-geometry then is the possibility of comparing small measuring rods at a distance. As Reichenbach points out, "Weyl's generalization of the theory of relativity ... abandons altogether the concept of a definite length for an infinitely small measuring-rod" (Reichenbach, 1920, 73; tr. 1965, 76), that is, it drops the hypothesis of global availability of the linear unit of measure. Reichenbach correctly observes that "[i]f, for instance, Weyl's generalization should turn out to be correct ... [t]hen the comparison of two small measuring rods at two different space points would also no longer contain the objective relation that it contains in Einstein's theory" (Reichenbach, 1920, 87; tr. 1965, 91; translation modified).

Reichenbach did not hide his reservations toward Weyl's idea "that mathematics and physics are but one discipline" (Reichenbach, 1920, 73; tr. 1965, 76); however, he had all the elements to understand that Schlick's attempt to save conventionalism by shifting the attention from rigid bodies to infinitesimal rigid rods simply misses the point. In Riemannian geometry, the choice of the unit rod or of the unit clock is of course arbitrary, but the ratio of the units is constant all over the manifold. This is a matter of fact and not of convention.

In the years between 1920 and 1929 Reichenbach played a fundamental role in defending and popularizing Einstein's theories (Hentschel, 1982), at the same time acquiring an admirable technical knowledge of their mathematical apparatus. Surprisingly, however, probably under the influence of Schlick, he abandoned his very convincing critique of geometrical conventionalism.

After an opportunity to have a first look at Reichenbach's book, Schlick immediately wrote to Einstein: "Reichenbach does not seem to me to be fair [nicht gerecht zu sein] toward Poincaré's conventionalism [Konventionslehre]" (Schlick an Einstein, 9.10.1920; CPAE 10, Doc. 171). Writing to Reichenbach some weeks later, Schlick tried to debunk Reichenbach's critique of conventionalism, arguing (erroneously) that Poincaré in his later writings included geometries of variable curvature in his approach (Reichenbach to Schlick 26.11.1920; Schlick and Reichenbach, 22).

Reichenbach answered, agreeing on Schlick's " and Einstein's point of view" [und Einsteins Standpunkt] that in principle one could choose between keeping relativity and abandoning Euclidean geometry or vice versa: "physics, however, makes the first decision ... you, and Poincaré, would say for the sake of simplicity [um der Einfachheit halber] ... But I have an instinctive refusal against this interpretation" (Reichenbach to Schlick 29.11.1920; Schlick and Reichenbach, 22). As is well known, Reichenbach very rapidly overcame his "instinctive disinclination" toward conventionalism, a fact that still puzzles historians (on this point see: Parrini, 2005). In the following years Reichenbach would describe Einstein's epistemological achievement precisely as the discovery that would have made it possible in principle for physics to make the second decision, that is, to get rid of non-Euclidean geometry by preserving gravitation as a real force.

\subsection{Einstein's Geometrie und Erfahrung}

The publication of the expanded form of Einstein's 1921 lecture Geometrie und Erfahrung (Einstein, 1921, tr. in Einstein, 1954, 232-246) probably played a major role in Reichenbach's "conversion". In the lecture, Einstein referred explicitly to Schlick's book on the theory of knowledge (Schlick, 1918), which he had read during a journey to Holland in October 1919 (cf. Howard, 1984, 620).

Following Schlick's method of implicit definitions (Schlick, $1918, \S 7)$, Einstein distinguishes between "purely axiomatic geometry" and "practical geometry", which "contains affirmations as to the relations of practically-rigid bodies" (Einstein, 1921, 6; tr. 1954, 235). Einstein even claims that, if "this view of geometry" as a "branch of physics", "had not served as a stepping-stone", he "should have been unable to formulate the theory of relativity" (Einstein, 1921, 6f.; tr. 1954, 235).

Einstein, however, famously recognized that Poincaré was "sub specie aeterni" right (Einstein, 1921, 8; tr. 1954, 236) when he denied that the physical behavior of rigid bodies can be used to establish whether the abstract geometry $(G)$ is true or false; in principle it is always possible to make rigid bodies agree with any kind of geometry we please by changing the physical laws $(P)$ that govern their behavior. Only the sum $G+P$, geometry plus physics, can be compared with experience (Einstein, 1921, 7-8; tr. 1954, 236).

The reference to Poincaré is at first sight quite puzzling. The concept of "rigid body" which lies at the basis of his philosophy of geometry is already quite problematic in Special Relativity, and becomes completely useless in a space of variable curvature, such as that of General Relativity (Friedman, 2002). Einstein was of course referring to "practical infinitesimal rigid rods [praktisch unendlich kleinen Massstabe]" (CPAE 7, Doc. 31, 271).

General Relativity presupposes that, if two of these rods "are found to be equal", i.e. have the same length, "once and anywhere, they are equal always and everywhere" (Einstein, 1921, 9; tr. 1954, 237). The same assumption must be made for clocks, that "when going at the same rate" in some place and time, "they will always go at the same rate, no matter where and when they are again compared with each other" (Einstein, 1921, 9f.; tr. 1954, 238) The name of Weyl is not explicitly mentioned by Einstein. However, Einstein's argument to support this claim is only a thinly veiled allusion to the "measuring-rod objection" against him: "The existence of sharp spectral lines - Einstein writes - is a convincing experimental proof of the above-mentioned principle of practical geometry" (Einstein, 1921, 9; tr. 1954, 238; my emphasis) 
Thus the reference to Poincaré in Einstein's Geometrie und Erfahrung has nothing to do with the classical problem of choosing among Euclidean and non-Euclidean sorts of Riemannian geometries. On the contrary, the very nature of Riemannian (or pseudo-Riemannian) geometry is at stake. The transportability of small measuring rods and atomic clocks - the fact that their relative lengths and rates are independent of their position — "is the ultimate foundation ... which enables us to speak with meaning of a metric in Riemann's sense of the word" (Einstein, 1921, 11; tr. 1954, 238; my emphasis).

As Einstein noticed in a note to an unpublished article intended for "Nature" (Grundgedanken und Methoden der Relativitätstheorie, in ihrer Entwicklung dargestellt, 1920), "this sort of equality" between rods and clocks, "an endurable, independent from the motion-prehistory [eine dauernde, von der Bewegungsvorgeschichte unabhängige], is "a crucial [massgebende] presupposition of the entire theory" (CPAE 7, Doc. 31, 280). Einstein accepted such a presupposition as an empirical fact that small rods of equal length will remain so after arbitrary separation. Weyl could however object that such a behavior could not be directly read off from observations, since two equal measuring rods, because of the influence of temperature, external forces etc., will likely not have the same length when reunited; Einstein's alleged fact was no more than an arbitrary stipulation.

With a little good will one can glimpse here some vague resemblance to Poincaré's conventionalist arguments against Helmholtz's empiricism. Poincaré was ready to "save" Euclidean geometry $(G)$, by changing the laws of physics $(P)$; Weyl claimed that it was always possible to maintain a non-Riemannian "aether-geometry" [Äthergeometrie], by blaming the "body-geometry" [Körpergeometrie] (Weyl, 1921a, 232) - the empirically observed Riemannian behavior of rods and clocks - on the physical mechanism of "adjustment". From this point of view, as Weyl put it in the 1921 edition of Raum-Zeit-Materie, geometry and physics "form an inseparable unity, something that should be kept in sight always as a whole" (Weyl, 1921c, 60).

The interpretation of Geometrie und Erfahrung is then a paradigmatic case where Beller's "dialogical" approach turns out to be indispensable. The text is easily misunderstood if one does not bear in mind that Einstein, in referring to Poincaré, was actually addressing Weyl's epistemological question without mentioning him (cf. Ryckman, 2005, §3.5).

It is instructive to pay attention to Einstein's phrasing. According to Einstein, Poincaré was sub specie aeterni right for the following reason: he had shown that solid bodies and clocks are not "irreducible elements" but "composite structures", which must "not play any independent part in theoretical physics" (Einstein, 1921, 8; tr. 1954, 236; my empahsis); in principle they should be constructed "theoretically from elementary concepts". However, according to Einstein, "in the present stage of development of theoretical physics" this is not possible, and "these concepts must still be employed as independent concepts" (Einstein, 1921, 8; tr. 1954, 237; my empahsis)

These are of course exactly the alternative attitudes toward the role of rods and clocks in General Relativity that Einstein, Weyl and Dällenbach had debated in public writings and in private correspondence some years earlier. Weyl still insisted on this point in a 1921 paper: Einstein's "measure-determination ... with help from measuring rods and clocks" can be accepted only to assure "a preliminary connection to experience"; in principle, however, the transportability (or non transportability) of rods and clocks should be regarded "as consequences of the developed theory" (Weyl, 1921b, 259-260).

In a paper published in the same year — addressing Reichenbach's 1920 critique directly — Weyl seems to regard precisely this point as the major "philosophical" implication of his attempt to unify gravitation and electricity (cf. Rynasiewicz, 2005):

From different sides, ${ }^{2}$ it has been argued against my theory, that it would attempt to demonstrate in a purely speculative way something a priori about matters on which only experience can actually decide. This is a misunderstanding. Of course from the epistemological principle [aus dem erkenntnistheoretischen Prinzip] of the relativity of magnitude does not follow that the "tract" displacement [Streckenübertragung] through "congruent displacement" [durch kongruente Verpflanzung] is not integrable; from the principle that no fact can be derived. The principle only teaches that the integrability per se must not be retained, but, if it is realized, it must be understood as the outflow [Ausfluß] of a law of nature (Weyl, 1921a, 475; last emphasis mine).

Weyl clears the ground of possible misunderstandings; his theory intended only to show that Einstein's assumption about the existence of rigid infinitesimal rods and ideal clocks is not logically necessary, but only a factual claim that General Relativity should be able to justify. There is little doubt that Einstein, in his 1921 lecture, was addressing precisely this challenge of Weyl's, recognizing it as inescapable sub specie aeternitatis, even if impossible to overcome sub specie temporis.

\subsection{A Parting of the Ways: Schlick's and Reichenbach's Misunderstanding of Einstein's Epistemology of Ge- ometry}

Isolated from the background of the dialogue with Weyl, Geometrie und Erfahrung seemed to confirm Schlick's philosophy of geometry beyond every hope. Not only did Einstein explicitly mention Schlick's 1918 book, but he also referred explicitly to Poincaré, apparently interpreting his own theory in the light of the 19th century debate about geometry. Schlick's famous Erläuterungen to Helmholtz's

${ }^{2}$ The reference is to Reichenbach, 1920 and Freundlich, 1920 
writing on geometry (Helmholtz, 1921) show how easily Einstein's formula $G+P$ could be integrated with Schlick's own conventionalism. Einstein had somehow reconciled the Helmholtz-Poincaré antithesis with a sort of Hegelian synthesis: one can determine the geometry of space empirically by assuming the conventional definition of rigidity of bodies that leads to the simplest physics (see e.g. Helmholtz, 1921, Schlcik's note 31).

In his recension of Einstein's Geometrie und Erfahrung, published on June, 3th 1921 on Die Naturwissenschaften, Schlick seems however to be aware that there was another problem about which Einstein was concerned:

It has been often noticed that there is no simple compelling reason to choose some physical bodies [Naturkörper] as rigid; we could use an arbitrary [beliebiges] system of geometry for the description of reality, if only we are ready to change at the same time the system of physics; only the totality, geometry plus physics, is determined forcefully by experience. Einstein recognizes the in principle irrefutable validity of this conception; however just as Poincaré admitted, that the economy of science forces us to choose, without hesitation, a determinate geometry (even if he thought it was the Euclidean one); similarly for Einstein in today's state of development of physics, it is inevitable to use [zugrunde zu legen] empirical physical bodies [Naturkörper] with determinate properties as rigid measuring rods in the measurement of the displacement possibilities [Lagerungsmöglichkeiten]; one should use those bodies which satisfy that condition which can be confirmed by the experience - that two measuring rods are always and overall equally long, if they once and somewhere were found as equal (a condition, which Weyl, as well-known, had tried to drop). Under this condition the axioms of practical geometry are pure empirical sentences that can be communicated through observation (Schlick, 1921, 435; my emphasis).

Schlick, of course, could appreciate Einstein's refinement of Poincaré's "economical" procedure of making an, in principle, arbitrary decision about which rods are rigid. However, by the end of the passage, Schlick also makes a more subtle point. He remarks that such rods must satisfy a "condition" (the ratio of the lengths of two rods is the same whenever they are compared) that it is "confirmed by experience", even if Weyl has "tried to drop it". Thus Schlick was aware of Einstein's measuring rod objection. Surprisingly, however, he did not seem to realize that precisely the epistemological status of this "condition" (whether it is only "confirmed by experience", or if it should be "derived from the theory"), was Einstein's main epistemological concern.

After all, Einstein mentions Schlick's own book at the very beginning of the Geometrie und Erfahrung, but does not mention the name of Weyl. Schlick could then have the legitimate impression that Einstein was pursuing a similar "Poincaréian" agenda, revolved on the classical prob- lem of the choice among Euclidean and non-Euclidean geometry. This misunderstanding can be appreciated even more clearly if one considers the following passage of Reichenbach's review article on philosophical interpretations of Relativity published in the same year:

[1] We have mentioned above, that the congruence of two tracts can be defined through transportation of a natural measuring-rod; however this is of course only a definition. It could also be defined in another way; for instance, a measuring rod after two juxtapositions becomes $1 / 2$ of its original length, after three $1 / 3$ etc. One obtains then a Riemannian geometry of different measuring-determination [Maßbestimmung]. The "change" of the measuring rods can in this case be interpreted as an effect of force, that in this way it is introduced in the definition [hinzudefiniert] [of a rigid rod]. Depending on the choice of the field of force, one gets a different geometry. For this reason material objects [materiellen Gebilde] do not define a single geometry, but a class of geometries; this is precisely the meaning of conventionalism. [2] However one must consider, that this class of geometries is in turn not arbitrary, but their validity is based on an axiom, that denotes an empirical fact [einen empirischen Tatbestand]; it is the assumption that two natural measuring rods, which can be brought to superposition once [die sich einmal zur Deckung bringen lassen], can be superposed again after they have been transported along different paths. In the validity of this axiom - we want to call it the axiom of the class of Riemannian geometries - lies an invariant characteristic of the possible geometry according to Einstein (Reichenbach, 1921, 365-366)

This passage is in my opinion extremely significant and deserves a careful reading. It shows precisely the point where Einstein and Logical Empiricists came to a misunderstanding:

[1] In the first part of the quote, Reichenbach refers to the classical problem of the choice among possible Riemannian geometries with different measure determinations. By arguing that a non-Euclidean geometry is equivalent to a Euclidean geometry with a field of universal forces, Reichenbach shows that the choice is arbitrary. This was the original problem discussed by Helmholtz and Poincaré, and adapted by Schlick in the new general-relativistic context: "According to the theory of relativity, the choice of a geometry is arbitrary; but it is no longer arbitrary once congruence has been defined by means of rigid bodies" (Reichenbach, 1921, 360; 1978, I, 38).

[2] In the second part of the passage, however, Reichenbach makes a different and, for our goals, more important consideration. Reichenbach notices that all Riemannian geometries (Euclidean or non-Euclidean) share a common property: they all presuppose that two measuring rods of the same length remain so if 
separated and brought together again. As Reichenbach correctly notices, this "axiom" — actually an empirical fact - defines the entire class of Riemannian geometries, that is of the "possible geometries according to Einstein".

It is not exaggerated to claim that here we have come to a sort of a parting of the ways: Reichenbach, in subsequent years, will base his philosophical interpretation of General Relativity on the conviction that Einstein was mainly concerned with problem [1]. It is not by chance that, still in the 1978 English translation of Reichenbach's paper, the pages on Weyl's theory were simply omitted as "of no historical importance" (Reichenbach, 1978, 38). However, as we have seen, it was precisely problem [2] which Einstein was addressing.

It must be emphasized that Reichenbach understood perfectly well the epistemological implications of Weyl's theory; he simply did not recognize them as the central issue of Einstein's reflections on geometry. Reichenbach in fact took back his previous objection "that Weyl wants to deduce physics from Reason, since Weyl has cleared up this misunderstanding" (Reichenbach, 1921, 367; the reference is to Weyl 1921a, 475). He had come to realize that Weyl did not want to impose on nature his purely infinitesimal geometry, but rather show that the transportability of rods and clocks - uncritically assumed by Einstein is actually not logically necessary:

Weyl took exception to the fact, that Einstein has simply condoned [einfach hingenommen] the univocal transportability of natural measuring-rods [eindeutigen Uebertragbarkeit natürlicher Maßstäbe]. He does not want to dispute the axiom of the Riemannian class for natural measuring rods; he wants only to urge that the validity of this axiom, being not logically necessary, "is understood as an outflow [Ausfluß] of a law of nature". I can only agree with this demand [Forderung]; it is the meaning of mathematics, that, by discovering more general possibilities, reveals the contingency of certain facts of experience [speziellen Tatbestände der Erfahrung als speziell kennzeichnet] and thus save Physics from simplification [Simplizität]. Admittedly, Weyl was able to explain the univocal transportability of natural measuring-rods only in a very incomplete way. But the only fact that he had tried to follow this path, regardless of its empirical correctness, was a genial advance [genialer Vorstoß] in the philosophical foundation of physics (Reichenbach, 1921, 367f.).

Here, Reichenbach sees the point clearly: Einstein assumed the Riemannian idea of measurement as a simple fact (einfach hingenommen): the "univocal transportability of natural measuring-rods" is an independent hypothesis which must be presupposed to assure the comparability of the predictions of the theory with reality. Weyl, by showing that another class of geometries where this assumption is dropped would be equally possible, had rightly asked for an explanation: the "univocal transportability of natural measuring-rods" must be derived from the theory - it must be a consequence of it.

Reichenbach provides an admirable presentation of the Weyl-Einstein controversy: Einstein's de facto Riemannian assumption vs. Weyl's requirement of its de iure justification in front of an equally feasible non Riemannianalternative. What Reichenbach seems to have missed is the fact that Einstein, by referring to Poincaré in his 1921 lecture, was addressing precisely this issue and not supporting a form of conventionalism à la Schlick. The original problem discussed by Poincaré, namely the choice within the class of possible Riemannian geometries was not at stake at all.

On the contrary, Reichenbach reneges his previous opinion that "the metric ... expresses an objective property of reality" and insists that "[t]his conception does not contradict conventionalism", which for Reichenbach is evidently implied in Einstein's formula $G+P$ : "Schlick must not be interpreted as saying that a certain metric has been prescribed; a metric emerges only after the physical laws have been established (the $P$ of Einstein's formula). One can also change the metric, provided one changes the laws of physics correspondingly" (Reichenbach, 1921, 356; tr. 1878, I, 34f.; my emphasis).

\subsection{Getting off Track: The Implementation of Reichen- bach's Conventionalism}

Reichenbach's 1922 paper, La signification philosophique de la théorie de la relativité (Reichenbach, 1922), the first comprehensive presentation of his new conventionalist approach to geometry and relativity, shows clearly that he, in the wake of Schlick, had come to interpret Einstein's reference to Poincaré, so to say, "literally": "The solution to the problem of space is therefore found only in this conception we call conventionalism, which goes back to Helmholtz and Poincaré" (Reichenbach, 1922, 40; tr. 2006 ; 135). The central problem for Reichenbach is precisely how to choose among different possible Riemannian geometries.

By introducing the idea of a non-detectable force of type $X$, such as gravitation, which causes uniform shrinkages and expansions in all materials, Reichenbach concludes that the main philosophical lesson of General Relativity is the following: "[t]he definition of congruence is ... arbitrary, and what is congruent in one geometry is not necessarily congruent in another" (Reichenbach, 1922, 33 ; tr. 2006; 127; my emphasis). It is worth noticing that Reichenbach makes an important remark here, although in passing: "This definition of congruence is arbitrary, but it is uni-vocal, and it entails that two rigid rods that are congruent at a point remain congruent at all points. This is an axiom that we can consider to be experimentally well confirmed" (Reichenbach, 1922, 35; tr. 2006; 129; my emphasis).

Reichenbach thus addresses the issue again that the univocality of the definition of "congruence" as such is only 
an experimental, and not a logical, truth. A non-univocal definition of congruence would be in principle legitimate: "This is the path that Weyl followed with perfect rigor; it cannot be said that a rod is equal in an absolute sense to one at another place, but that we can dispose altogether with the arbitrary process of the comparison of rods" (Reichenbach, 1922, 41; tr. 2006; 136).

Reichenbach seems here to confuse different issues. There is actually no univocal definition of congruence of bodies in Riemannian manifolds of variable curvature, as Reichenbach himself had rightly noticed in 1920. In such spaces, in fact, the congruence of bodies is not independent of position: two plane figures which are congruent on the equator of an egg-shaped surface cannot be reproduced at the pole. In all Riemannian manifolds, however, there is an arbitrary, but univocal definition of the unit of length: two small rods of equal lengths in a place will still have the same length wherever they are compared.

The fact that the univocality of the definition of a unit of length was only "experimentally well confirmed" was precisely Einstein's main and only epistemological concern that emerged in the discussion of Weyl. The fact that Reichenbach did not appreciate this point is nothing but stunning. Reichenbach, in fact, explains it with usual accuracy in his more technical 1924 Axiomatik der relativistischen Raum-Zeit-Lehre (Reichenbach, 1924, tr. Reichenbach, 1969).

As we have seen, using light rays $d s^{2}=0$, one can determine the value of $g_{\mu \nu}$ up to a scale factor: "then every metric $g_{\mu \nu}^{\prime}$ which results from $g_{\mu \nu}$ through multiplication by a scalar field $\lambda\left(x_{1}, \ldots, x_{4}\right)$ will satisfy these axioms, since in it the lines $d s^{2}=0$ satisfy the same equation" (Reichenbach, 1924, 120; tr. 1969, 151). As Reichenbach notices, if "the light geometry furnishes only the quotient $\frac{g_{\mu \nu}}{g_{\mu \nu}^{\prime}}=q_{\mu \nu}$, then "[f] or the determination of the absolute values of the $g_{\mu \nu}$ in the gravitational field, however, we need material things, either natural clocks or rigid rods" (Reichenbach, 1924, 122; tr. 1969, 170).

Thus Reichenbach shows very clearly that we need rods and clocks only in order to force the parameter $\lambda$ to be constant:

One can now imagine the construction of the metric $g_{\mu \nu}$. If any coordinate system $K$ is given for the world, then point events of the distance $d s^{2}=+1$, $d s^{2}=0, d s^{2}=-1$ can be produced by means of rigid rods and clocks permanently at rest in $K$. The world will thus be interspersed with unit mesh points. The functions $g_{\mu \nu}$ are to be determined in such a way that with the chosen coordinates $d s= \pm 1$ or 0 , respectively, for all mesh points. If merely the quotients $q_{\mu \nu}$ are to be constructed, light signals alone suffice. ... Only in order to make the unit of length (or the unit of time) of the various systems $K^{\prime}$ equal are transportable rigid rods (or clocks) needed. The significance of material things becomes clear: they bring about $a$ comparison of the units at different points. This comparison cannot be achieved by means of light signals; for all other purposes light is sufficient (Reichenbach, 1924, 120; tr. 1969, 151; my emphasis)

Thus Reichenbach is completely clear about the fact that only in order to assure the reproducibility of the unit of length we need the rigid rods and clocks. If the ratios of the $g_{\mu \nu}$ is determined by using light signals, then clocks and rods serve to determine the factor $\lambda$. The requirement that two small rigid rods are congruent at a point and remain congruent at all points is identical to the assumption that scale factor $\lambda$ is constant. This property characterizes the class of Riemannian geometries as such and says nothing about the possibility of making a choice within this class. As we have seen, the ratio of two of such small rods and therefore also their ratio $=1$, their equality — is an absolute constant in General Relativity, and thus it is not subjected to any conventional stipulation.

The relevant epistemological problem Einstein was forced to consider after his debate with Weyl was precisely that of the status of such a transportability of rods and clocks in General Relativity. Again one has only to follow Reichenbach's exposition:

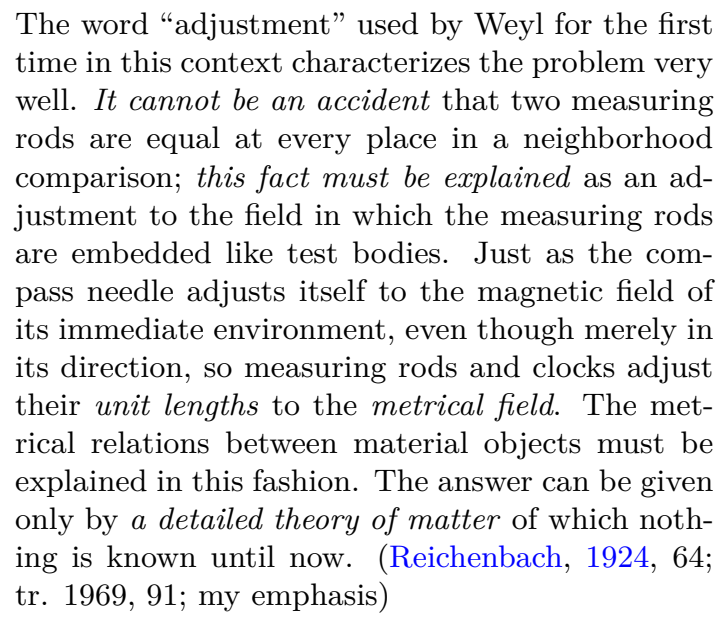

Here, once again, Reichenbach explains the vexed question eloquently: "it cannot be an accident that two measuring rods are equal at every place in a neighborhood comparison"; Weyl had therefore required "this fact must be explained". Precisely like Einstein, however, Reichenbach claims that we do not have a "detailed theory of matter" from which one can deduce such a behavior of our rods. It is thus almost incomprehensible that Reichenbach failed once again to realize that Einstein, in his 1921 lecture, was addressing this issue exactly and not renewing the empiricism/conventionalism debate between Helmholtz and Poincaré.

\subsection{Einstein's Reflections on Philosophy of Geometry be- tween 1923 and 1926}

Schlick's 1925 second edition of his Allgemeine Erkenntnislehre confirms even more clearly that the reading of Einstein's lecture on the background of the HelmholtzPoincaré controversy prevailed among Logical Empiricists 
(Schlick, 1925, 326). Einstein's reflections on geometry published at about the same time, however, clearly suggest that Einstein's reference to 19th century conventionalism cannot be interpreted literally, but have to be inserted in its proper "dialogical" context.

In his delayed lecture for his 1921-22 Nobel prize delivered to the Nordic Assembly of Naturalists at Gothenburg in July 1923 Einstein had famously insisted that, as composite atomic systems, rigid bodies cannot be used to verify the laws of nature, for these very same laws should in principle account for their rigidity (D'Agostino, 2000, 241f.). According to Einstein, "it would be logically more correct to begin with the whole of the laws" (Einstein, 1923, 3; my emphasis) and not with an "artificially isolated part" such as rods and clocks (Einstein, 1923, 3; my emphasis).

In a 1924 recension (Einstein, 1924b) of a book by a minor Neo-kantian, Adolf Elsbach (Elsbach, 1924), to which attention has been drawn recently (Howard, 1990, 2010), Einstein distinguishes two different "standpoints" on the question of "whether one grants reality to the practicallyrigid body": according to Standpoint $A$, geometry "contains assertions about possible experiments"; on the contrary, according to Standpoint B, "only geometry with physical sciences taken together" can be compared with experience (Einstein, 1924b, 1690f.). It is not hard to see here once again the opposition between Einstein's and Weyl's epistemological stance.

Even more important, in a paper published one year later, Nichteuklidische Geometrie und Physik (Einstein, 1925), Einstein expressly attributed standpoint $A$ to Poincaré, whereas standpoint $B$ to Helmholtz:

In order to see the matter clearly, one must consistently adopt one of two points of view. $[A]$ In the first, one holds that the "body" of geometry is realized in principle by rigid bodies in nature, provided that certain conditions are met regarding temperature, mechanical strain, etc.; this is the point of view of the practical physicist. In this case, the "distance" of geometry agrees with a natural object and thereby all propositions of geometry gain the character of assertions about real bodies. This point of view was especially clearly advocated by Helmholtz, and we can add that without him the formulation of relativity theory would have been practically impossible. $[B]$ In the other point of view, one denies in principle the existence of objects that agree with the fundamental concepts of geometry. Then geometry by itself would include no assertions about objects of reality, only geometry taken together with physics. This point of view, which may more complete for the systematic representation of a finished physics, was expounded particularly clearly by Poincaré, from this standpoint, the entire content of geometry is conventional; which geometry is preferable depends on how "simple" physics can be made by using geometry to agree with experience (Einstein,
1925, 253, tr. in Pesic, 2007, 161)

Again Einstein is evidently not referring to finite rigid bodies here; in a space of variable curvature "the possible contact-displacements [Berührungslagerungen] of practically rigid bodies would be different in different cases, conditioned by the influence of the circumstances [MilieuEinflüssen]" (Einstein, 1924a, 86); there is no unique set of congruence relations, so that it would not make sense to decide once and for all which tiles of space are congruent, as Helmholtz's and Poincaré's philosophy of geometry would require. Einstein is referring, of course, to the "the possible disposition of infinitely many, infinitely small rigid bodies [Lagerungsmöglichkeiten für unendlich viel unendlich kleine starre Körper]" (Einstein, 1925, 253, tr. in Pesic, 2007, 161). The local Euclidean behavior of such rods cannot be extended "over finite regions" of space (Einstein, 1925, 253, tr. in Pesic, 2007, 161), at least if we attribute the same length to one of such rods in all positions and in every orientation.

Einstein's reference to Helmholtz and Poincaré, then, is nothing more then a "rhetorical device" which actually serves to address Weyl's challenge. Einstein adopted the "Helmholtzian" standpoint $A$, accepting provisionally as an independent fact that there are rigid infinitesimal rods, whose length would be the same under all circumstances. Weyl followed a "Poincaréan" standpoint $B$, by arguing that such a behavior of infinitesimal rods cannot be observed, but it is at most a convenient stipulation.

The problem with which Einstein was concerned was then completely detached from the historical dialogue between Helmholtz and Poincaré, whose names are used as mere labels to identify two abstract philosophical positions: "consistent thinkers - as Einstein put it in the entry "space-time" for the 13th 1926 edition of the Encyclopedia Britannica - considered it preferable to allow the content of experience [Erfahrungsbestände] to correspond to geometry and physics conjointly" (Einstein, 1926, 609). Einstein, however, is still convinced that "it would not be advisable to give up the first view, from which geometry derives its origin" - i.e. the interpretation of geometry as science of the behavior of rigid bodies —- "an abstraction that is well rooted in the laws of nature" (Einstein, 1926, $609)$.

Logical Empiricists not only failed to appreciate that Einstein supported standpoint $A$ only provisionally — as it is usually claimed in literature. They never understood the very problem to which the alternative referred: the reproducibility vs. "not-reproducibility of the gauge-unit [Nichtreproduzierbarkeit der Eicheinheit]", as Fritz London put it (London, 1927, 187). Weyl's theory at that time was losing most of its convincing power. As is well known, however, London's suggestion to replace the scale factor in the original theory with a phase factor would make Weyl's "gauge invariance" one of the central feature of 20th century physics (Yang, 1986; O'Raifeartaigh and Straumann, 2000). 


\section{Reichenbach's Relativity of Geometry}

Reichenbach was of course completely aware of the problem that "non-reproducibility of the gauge-unit" was a relevant issue. He discussed it again as early as in $\$ 4$ of his classical semi-popular Philosophie der Raum-ZeitLehre, which he had already finished in 1926 (but will be published as Reichenbach, 1928, tr. Reichenbach, 1958). As is well known, according to Reichenbach, whereas the definition of the concept of the unit of length is a conceptual definition, the choice of the interval that serves as a unit of length is a coordinative definition - a definition which coordinates an abstract concept with a "piece of reality", such as the meter standard in Paris (cfr. Shapiro, 1994). Reichenbach, however, adds this significant consideration:

After this solution of the problem of the unit of length, the next step leads to the comparison of two units of lengths at different locations. ... Assume two measuring rods which are equal in length. They are transported by different paths to a distant place; there again they are laid down side by side and found equal in length ... it is an observational fact [beobachtbare Tatsache], formulated in an empirical statement [Erfahrungssatz], that two measuring rods which are shown to be equal in length by local comparison made at a certain space point will be found equal in length by local comparison at every other space point, whether they have been transported along the same or different paths ... The physical fact makes the convention univocal (eindeutig), i.e., independent of the path of transportation. The statement about the univocalness (Eindeutigkeit) of the convention is therefore empirically verifiable and not a matter of choice. One can say that the factual relations holding for a local comparison of rods, though they do not require the definition of congruence in terms of transported rods, make this definition admissible. Definitions that are not unique are inadmissible in a scientific system (Reichenbach, 1928, 24f.; tr. 1958, 17; my emphasis; trasnlation slightly modified).

Reichenbach is then aware that it is only " a matter of fact that our world" admits a "univocal" definition of the unit of measure (Reichenbach, 1928, 25; tr. 1958, 17); it is only for the "factual relations holding for the behavior of rigid rods" (Reichenbach, 1928, 27; tr. 1958, 17). The choice of a rod as the standard unit of length is arbitrary, is a matter of definition or convention, but it is a matter of fact that two of such unit rods are equal everywhere when compared,. Reichenbach, like Einstein, accepted this "fact" and elevated it as a very condition for a "coordinative definition" (Reichenbach, 1928, 27; tr. 1958, 17).

However, the question of the univocality of the definition of the unit of measure is clearly not the central problem for Reichenbach. Rather, he moves quite abruptly to the question of the definition of the "congruence of bodies" (Reichenbach, 1928, §5), which is at the basis of his famous "relativity of geometry" (Reichenbach, 1928, §8). Reichenbach famously claims that the relativity of geometry "is essentially the result of the work of Riemann, Helmholtz, and Poincaré" (Reichenbach, 1928, 48; tr. 1958, 35), who - in Reichenbach's view - first recognized that the geometry of space, whether Euclidean or non-Euclidean, rests on a conventional definition of which bodies are rigid. Einstein simply applied this approach to physics.

Of course, as Reichenbach himself noticed, even if only by the end of the book, that "there are no rigid bodies" in General Relativistic space-time; the concept of rigidity "loses its definiteness in fields in which the adjustment of measuring instruments is not uniform" (Reichenbach, 1928, 302; tr. 1958, 264). Reichenbach, however, believed that he still had an "ace in the hole"; in General Relativity the coordinative definition does not concern finite bodies, but rather "infinitesimal measuring instruments" (Reichenbach, 1928, 302; tr. 1958, 264; my emphasis). As it has been pointed out (Torretti, 1983, 239f.), however, Reichenbach's approach is hardly compatible with his conventionalism, as it paradoxically emerged from his own account.

Reichenbach explains with the usual clarity that in order to perform measurements, we "carry around infinitesimal measuring rods" (Reichenbach, 1928, 285; tr. 1958, 249), which we assume to be unit rods. Riemann's geometry assumes that the laws of disposition of such rods more closely approach those of Euclidean geometry the smaller the dimensions of the considered region of space become. Rods are progressively more rigid to the extent that they are smaller. If our rods do not show such a Euclidean behavior, as Reichenbach observes, "we would assume, therefore, that the unit rod was not chosen sufficiently small and would claim that the axiom would be satisfied if the rod were shortened" (Reichenbach, 1928, 286 ; tr. 1958,250 ). So the rigidity of rods is evidently not stipulated arbitrarily, but checked under the presupposition that space is Euclidean in small domains (Torretti, 1983, 239f.).

After having determined a rigid unit rod according to this procedure, the geometry of space around a point can then be found empirically, under the presupposition "that $d s^{2}$ be equal to 1 for the same $g_{\mu \nu}$ and any direction of the rod rotated in $P$ the $g_{\mu \nu}$ will be determined uniquely [eindeutig]" (Reichenbach, 1928, 285; tr. 1958, 249) (up to a coordinate transformation). If in small regions of space $g_{\mu \nu}$ are necessarily constant, over larger regions of space, we might discover that, by combining the coordinate differentials according the Euclidean formula, "the resulting $d s^{2}$ is by no means equal to 1 " (Reichenbach, 1928, 285; tr. 1958, 249). We would then be forced to introduce "the corrections factors $g_{\mu \nu}$ ", so "that the $g_{\mu \nu}$ thus obtained will satisfy the condition $d s^{2}=1$ for all positions of the unit rod" (Reichenbach, 1928, 286; tr. 1958, 250). Other observers in other regions of spacetime will the adopt the same measurement procedure, and they will also find the value of the $g_{\mu \nu}$ in their neighborhood. Riemannian ge- 
ometry presupposed that not only the ratio $g_{\mu \nu}=\lambda g_{\mu \nu}^{\prime}$, but the value of the constant $\lambda$ can be determined after all observers have come to agree on the unit of measure to use: "Through experiment we discover at every point those numbers $g_{\mu \nu}$ by which the coordinate differentials must be multiplied in order that the interval will equal 1 " (Reichenbach, 1928, 287; tr. 1958, 251).

This procedure can of course be easily extended to the case of a pseudo-Riemannian manifold of General Relativity, where "[t]he coordinative definition of congruence is again given in terms of clocks, rods, and light rays" (Reichenbach, 1928, 287; tr. 1958, 251). If the field equations predict a certain value of the $g_{\mu \nu}$ (up to a coordinate transformation) - let's say the Schwarzschild solution for spatial spherical symmetry — we could then verify if the predictions of the theory were "true" or "false", using light rays "as a realization of $d s^{2}=0$, and infinitesimal clocks and rods "as realizations of $d s^{2}= \pm 1$ " (Reichenbach, 1928, 287 ; tr. 1958, 251)

This is of course perfectly true, but it is also worth clarifying that this is not "a coordinative definition of congruence" in the sense of Helmholtz and Poincaré. As we have seen, the definition of "congruence of bodies" is not univocal in General Relativity; there is no single set of congruence relations; the very idea of making a unique conventional choice among different incompatible sets does not even make sense (Friedman, 1995). As Reichenbach had explicitly shown in 1924, rods and clocks only serve to determine the choice of the linear unit of measure, which in General Relativity is regarded as arbitrary, but univocal.

Usually units of measure are defined with reference to an individual standard, such as the meter standard preserved in Paris; of course, it is more useful when standards are defined through class terms, for instance "the linear unit may be defined by means of the wave length of the red cadmium line. ${ }^{3}$ The similarity of all cadmium atoms is used for this purpose, and there is no need to store a special unit at a definite location" (Reichenbach, 1929a, 30; tr. 1978, I, 161). The unit of measure can easily be replicated all over the manifold.

Even though, in Reichenbach's parlance, this is a "coordinative definition", clearly it does not concern the choice among Euclidean and non-Euclidean geometry. As we have seen, by coordinating $d s^{2}=-1$ with another atom, let's say an atom of sodium with a yellow emitting line, all lengths would be changed by a scale factor, but their ratios would remain unchanged. Thus the spacetime metric would remain the same, being defined only up to a constant scale factor.

Rather, the real philosophical problem lurks in the phrase, "there is no need to store a special unit at a definite location". The stability of atomic spectra shows that there

\footnotetext{
${ }^{3}$ In 1927, the International Conference on Weights and Measures redefined the meter in terms of a red cadmium spectral line $(1 \mathrm{~m}=$ $1,553,164.13$ times the wavelength of the $6436.4696 \AA$ cadmium red line).
}

is a class of similar objects that can be used to reproduced the unit of measure in different positions (Reichenbach, 1928, 355). According to Reichenbach, the "objects in question are similar is, of course, not established by definition, but is a fact that must be discovered" (Reichenbach, 1929a, 30; tr. 1978, I, 161; my emphasis).

To find an explicit discussion about the nature of this factual presupposition we must wait, however, for the long appendix on Weyl's theory (Reichenbach, 1928, 331-373):

\begin{abstract}
The foundation of Riemannian space [is that] two measuring rods which are found equal, when they are compared near to each other, will be again equally long if they are transported through different ways in another place; the same must be assumed for the unit clocks ... We have noticed above that such a definition [of equality of length] is only for this reason possible, because, the measuringbodies posses the mentioned special property [Vorzugseigenschaft]; if the measuring rods would have different length every time they met, the usual definition of congruence would not be possible. The mentioned property is not a means to obtain a knowledge of the equality of tracts ... but it is a necessary condition for a definition of congruence distinguished by this special property [Vorzugseigenschaft] (on this topic see $\S 4$ ) (Reichenbach, 1928, 332)
\end{abstract}

Reichenbach refers here explicitly to the $\S 4$ of his book where he discussed precisely the "univocality" of the definition of "equality of tracts". The fundamental question is then the following. "What would happen if the measuring rods would not posses the mentioned special property [Vorzugseigenschaft]?" (Reichenbach, 1928, 332). Reichenbach surprisingly consigned this question to the appendix of his book (which will not even be translated into English). However, it is not at all exaggerated to argue that this was the core problem of Einstein's reflections on geometry. Einstein had assumed the "Vorzugseigenschaft" of rods and clocks as an independent "fact"; Weyl had showed that the alleged fact is at most a "stipulation".

Reichenbach explains very minutely the technical details of Weyl's theory in the appendix (cf. Coffa, 1979). A brief summary can be found, for instance, in a paper of 1929 discussing Einstein's new geometry based on distantparallelism (on this topic cf. Sauer, 2006):

Weyl noticed that Riemannian geometry contains a special presupposition: two measuring rods which, if compared next to each other are equal, are always equal, if, after having been transported through different ways, are compared again. Weyl asked, whether it would be possible to conceive more general geometrical relations, in which the rods change their length in dependence from the way along which they are transported; he found a mathematical procedure which allows to formulate this case ... In this way he produced an extension of the Riemannian space-type of undeniable mathematical meaning, which he however wanted to apply 
also to the physical problem just described [the unification of gravitation and electricity] ... such a theory, however, had to be abandoned for physical reasons; the effect of the electrical field on transported clocks [predicted by the theory]* was absent.

* One just has to think of an atomic clock, e.g. the rotating electron system of the atom, whose eventual change in frequency found expression in the emitted spectral lines. (Reichenbach, 1929b, 121)

Of course, Reichenbach had good reasons to consider Weyl's theory as discredited (see for instance Weyl, 1929). Reichenbach, however, does not seem to realize that, when Einstein refers, rather sporadically, to Helmholtz and Poincare he alludes exclusively to this "special presupposition" of Riemannian geometry, i.e. the "Riemannian method of space-measurements" (Reichenbach, 1929b, 121) of which Weyl had shown the contingency by introducing "a more richer geometry then the Riemannian one" (Reichenbach, 1929b, 121)

\section{Einstein vs. Reichenbach. From the Real to the Imaginary Dialogue}

As is well know, Einstein's esteem for Reichenbach was so high that in 1926 he managed, together with Max Planck, to create a teaching position for him in "natural philosophy" at the University of Berlin, where Reichenbach remained until the 1930s (Hecht and Hoffmann, 1982). Nevertheless, Reichenbach's famous doctrine of the "relativity of geometry" shows that surprisingly he was unable to appreciate that Einstein's reference to the dialogue between Helmholtz and Poincaré was little more than an homage and surely not the exposition of a philosophical program.

Reichenbach's attempt to name Einstein's theory as the heir of the Riemann-Helmholtz-Poincaré line was doomed to failure. Reichenbach completely neglected the grouptheoretical implications of Helmholtz and Poincaré's work (Friedman, 1995) - implications on which other philosophers such as Ernst Cassirer insisted (Cassirer, 1938, 1944, 1950, I, ch. 2; written in 1930s). Most of all he did not appreciate the fact that Riemann's work evolved along a different non-geometrical tradition - mainly in the work of Christoffel, Ricci and Levi-Civita (Reich, 1994) — whose geometrical significance was rediscovered only after General Relativity by Levi-Civita himself (Reich, 1992). The philosophical problem raised by Weyl's theory, which Einstein was actually addressing, can be understood exclusively within this tradition.

However, Reichenbach could certainly not be blamed for having lost track in following Einstein's complex "dialogical" network. Einstein resorted to the Logical Empiricists' favorite language of "coordination" to address Weyl's holistic challenge, and at the same time presented his dispute with Weyl as a novel version of the dialogue between Helmholtz and Poincaré. Behind Einstein's dialogical strategy probably lurks the exigence of extrapolating two general epistemological attitudes toward the geometry-physics relationship - the standpoints $A$ and $B$ of the Elsbach-review — from their more technical counterparts.

Einstein conformed to a sort of "double truth doctrine" all of his life. On the one hand, he never abandoned the conception of geometry as an investigation of "local relations of idealized corporeal objects" (Einstein, 1930c, 173; tr. in Pesic 2007, 173; see also Einstein, 1930a,b); geometry is "the science of laws governing the mutual position of practically rigid bodies" (Einstein, 1934, 227). é,In Physik und Realität (Einstein, 1936), Einstein even defined as a "fatal error [verhängnisvolle Irrtum]" the fact that this conception of geometry "has fallen into oblivion" (Einstein, 1936, 321, tr. 356).

On the other hand, Einstein candidly admits that, in General Relativity, such an assumption is not completely consistent. This is particularly clear in a famous passage of his Autobiographical notes (written in 1946, but published as Einstein, 1949a):
One is struck [by the fact] that the theory (ex- cept for the four-dimensional space) introduces two kinds of physical things, i.e., (1) measuring rods and clocks, (2) all other things, e.g., the electro- magnetic field, the material point, etc. This, in a certain sense, is inconsistent; strictly speaking measuring rods and clocks would have to be repre- sented as solutions of the basic equations (objects consisting of moving atomic configurations), not, as it were, as theoretically self-sufficient entities. However, the procedure justifies itself because it was clear from the very beginning that the postu- lates of the theory are not strong enough to deduce sufficiently complete equations from them for phys- ical events sufficiently free from arbitrariness, in order to base upon such a foundation a theory of measuring rods and clocks. If one did not wish to forego a physical interpretation of the co-ordinates in general (something which, in itself, would be possible), it was better to permit such inconsis- tency - with the obligation, however, of eliminat- ing it at a later stage of the theory. But one must not legalize the mentioned sin so far as to imagine that intervals are physical entities of a special type, intrinsically different from other physical variables (Einstein, 1949a, 59; my emphasis).

Einstein's epistemological preoccupations are then consistent with those that he had expressed in the past. In the final "Remarks Concerning the Essays Brought together in this Co-operative Volume" (finished in 1948) he emphasizes the following points:

1. General Relativity assumes that there is "such a thing as a natural object which incorporates the "naturalmeasuring-rod' independently of its position in fourdimensional space"; in particular "a spectral line" is 
"considered as a measure of a 'proper time' (EigenZeit) $\left(d s^{2}=g_{i k} d x_{i} d x_{k}\right)$ ". This point of view "made the invention of the General Theory of Relativity psychologically possible, however this supposition is logically not necessary" (Einstein, 1949b, 685; may emphasis).

2. This assumption is then not definitive: Only "[if] one disregards quantum structure, one can justify the introduction of the $g_{i k}$ 'operationally'", referring to "the existence of an arbitrarily sharp optical signal" (Einstein, 1949b, 686, my emphasis). We do not have at this time "a complete theory of physics as a totality" in which "the objects used as tools for measurement do not lead an independent existence alongside of the objects implicated by the fieldequations" (Einstein, 1949b, 686, my emphasis).

The echo of the debate with Weyl and Dällenbach (cf. §3) in the late 1910s can still be heard in these passages written thirty years later. The constancy of atoms gives physical meaning to the mathematical invariant $d s$; within General Relativity this remains an unverified assumption, which, in a later stage of the development of physics, should be derived from the theory.

As is well known, Einstein used exactly this form of holism à la Weyl against Reichenbach in the final "Remarks" (Einstein, 1949b). Interestingly, Einstein again resorts to the opposition between Helmholtz and Poincaré to display the epistemological implications of the points 1. and 2.: "Is a geometry ... verifiable (viz., falsifiable) or not? Reichenbach, together with Helmholtz, says: Yes, provided that the empirically given solid body realizes the concept of "distance" Poincaré says no and consequently is condemned by Reichenbach" (Einstein, 1949b, 677f.).

In his contribution to the volume, Reichenbach (Reichenbach, 1949), who Einstein praises for "the precision of deductions" and "the sharpness of his assertions", namely rehearsed his neo-conventionalist doctrine: after a definition of congruence has been chosen under the criterion that it does not imply redundant physical forces, then geometry of physical space (Euclidean or non-Euclidean) can be verified empirically using rods and clocks. Einstein famously replied by imagining a dialogue between "Reichenbach" and "Poincaré" (Einstein, 1949b). By the end of the dialogue an unidentified "Non-Positivist" bursts upon the scene. The passage is famous, but it is worth repeating again:

Non-Positivist: If, under the stated circumstances, you hold distance to be a legitimate concept, how then is it with your basic principle (meaning = verifiability)? Do you not have to reach the point where you must deny the meaning of geometrical concepts and theorems and to acknowledge meaning only within the completely developed theory of relativity (which, however, does not yet exist at all as a finished product)? Do you not have to admit that, in your sense of the word, no "meaning" can be attributed to the individual concepts and assertions of a physical theory at all, and to the entire system only insofar as it makes what is given in experience "intelligible?" Why do the individual concepts which occur in a theory require any specific justification anyway, if they are only indispensable within the framework of the logical structure of the theory, and the theory only in its entirety validates itself? (Einstein, 1949b, 678; my emphasis)

By substituting a non-Positivist for Poincaré, Einstein had probably come to realize that such a holism was not really implied by Poincaré's conventionalism, being rather similar to Pierre Duhem's epistemological stance (Grünbaum, 1963a, 133; for the historical plausibility of this hypothesis, see Howard, 1990).

The holism of the non-Positivist, however, clearly resembles that of Weyl's philosophy of geometry. In his 1949 English-augmented translation (Weyl, 1949) of his 1927 monograph Philosophie der Mathemaik und der Naturwissenschaft (Weyl, 1927), Weyl explicitly argues that geometry and physics can only be "put to the test as a whole" (Weyl, 1949, 134). Weyl's objections to Einstein were still vivid thirty years after their original dispute had started. It must be kept in mind that Einstein and Weyl were colleagues for nearly twenty years in Princeton. In an "Appendix" of the book Weyl still insisted on the fact that "the behavior of rods and clocks" should in principle come out "as a remote consequence of the fully developed theory" and not be used to measure "the fundamental quantity $d s^{2} "$ (Weyl, 1949, 288).

Beller's dialogical method once again becomes very useful. Einstein addressed several interlocutors at one time, interweaving different real dialogues in an imaginary one, in which, moreover, contenders from disparate epochs appear. Einstein's dialogical web it is then not easy to disentangle. Reichenbach, after all, could have gotten the impression that Einstein was attributing to him a view of the empirical character of geometry not unlike that which Einstein himself had used as a stepping-stone (and that he still defended in a 1951 letter to the Australian student Leonard Champion as reported by Stachel, 1989). However, it is quite clear that Einstein was addressing Weyl's objection; the apple of discord was once again "the existence of an arbitrarily sharp optical signal", that can be used to reproduce the unit of time at distance.

However, Logical Empiricists clearly still did not grasp this point. It is sufficient to consult their reviews of Schilpp's volume which came up immediately after its publication (Frank, 1949; Nagel, 1950). Similarly in his 1951 response to "Professor Einstein['s]" "witty defense of conventionalism" (Reichenbach, 1951, 135), Reichenbach simply rehearsed once again his neo-conventionalist position, entirely based on the question of establishing whether the choice among Euclidean and non-Euclidean geometries depends on an empirical or conventional definition of congruence. It is rather ironic that Reichenbach argued "that there can be no differences of opinion between mathemati- 
cal philosophers if only their opinions were clearly stated" (Reichenbach, 1951, 135). The lifelong dialogue that he had with Einstein, which traversed thirty years of their professional careers, seems to have been based on a complete misunderstanding.

\section{Conclusion: A Dialogue of the Deaf}

Modern philosophy of science is highly indebted to the dialogue between Einstein, Schlick and Reichenbach on the role of rods and clocks in General Relativity. Through this dialogue, Logical Empiricism made its first steps as a philosophical movement, which, especially in its "American incarnation" dominated 20th century philosophy of science, before stiffening into an over-simplified straw-man version that only recent historical literature has began to call into question.

However, this dialogue was surprisingly a dialogue of the deaf. The reason is disarmingly simple: Logical Empiricists were concerned with the question of making a choice within the class of Riemannian geometries. Einstein was rather worried by the legitimacy of the assumption that characterizes the class of Riemannian geometries as such, as included in the larger class of geometries put forward by Weyl. Logical Empiricists knew the latter issue perfectly well, but they never realized that Weyl was the interlocutor Einstein was actually addressing. In turn, Einstein never felt compelled to set things straight, so the illusion persisted that the subject of the debate revolved around the conventional vs. empirical choice between Euclidean and non-Euclidean geometries. This turned out to be a "a stubbornly persistent illusion", which was dragged along into successive famous debates, e.g. the GrünbaumPutnam controversy (Grünbaum, 1963b; Putnam, 1963; Grünbaum, 1968b,a, ch. III).

As Grünbaum's "Riemann-Poincaré principle of the conventionality of congruence" shows (cf. Giedymin, 1982), the misunderstanding between Einstein and Logical Empiricists is the result of what may be called a collision of mathematical traditions (Norton, 1999): The "Helmholtzian" tradition, which presupposes the "existence of bodies independent of position" and the "Riemannian" tradition, which started from the much weaker presupposition that "length lines is independent of position" (Freudenthal, 1956; Torretti, 1978, 1999).

Helmholtz's geometrical point of view was developed mathematically mainly by Sophus Lie (starting from Lie, 1886) in the theory of a continuous group of transformations (Lie, 1893), and was brought to its epistemological consequences by Poincaré (Friedman, 1995; Heinzmann, 2001 and others). Riemann's geometrical insight, however, remained dormant; it was mainly developed nongeometrically by Rudolph Lipschitz (Lipschitz, 1869), Christo fel, and Ricci in the form of a theory of differential invariants (Ricci-Curbastro, 1883, 1886, 1888, 1889), later systematized into the so-called "absolute differential calculus" (Ricci-Curbastro, 1892; Levi-Civita and Ricci-Curbastro,
1900), of which General Relativity, by Einstein's own account, was the "triumph" (Reich, 1994). The geometrical implications of Riemann's revolutionary approach were rediscovered only after the appearance of General Relativity, by, among others, Levi-Civita and Hessenberg, and appeared in radicalized form in Weyl "gauge invariance" principle.

Einstein's assumption that the relative length of rods does not depend on their pre-history is comprehensible only in the context of the "Riemannian" tradition. Logical Empiricists not only completely neglected the grouptheoretical implications of Helmholtz's approach, but most of all did not accord sufficient attention to the role that Riemann and his successors had played in the history of the epistemology of geometry. Neglecting the distinction between the Riemannian and the Helmholtzian tradition in the history of the philosophy of geometry, in an attempt to furnish "a Helmholtzian approach to space and time" (Darrigol, 2007) confuses, not unlike the Logical Empiricists, the problem of the comparability of the length lines at a distance with that of the congruence of bodies (Torretti, 1983, 238f.).

\section{References}

Bell, J. L. and H. Korté (2011). Hermann Weyl. In E. N. Zalta (Ed.), The Stanford Encyclopedia of Philosophy (Spring 2011 ed.).

Beller, M. (1999). Quantum Dialogue : the Making of a Revolution. Science and its Conceptual Foundations. Chicago: University of Chicago Press.

Born, M. (1909). Die Theorie des starren Elektrons in der Kinematik des Relativitätsprinzips. Annalen der Physik 11, 1-56.

Born, M. (1910). Über die Definition des starren Körpers in der Kinematik des Relativitätsprinzips. Physikalische Zeitschrift 11 , 233-234.

Cassirer, E. (1938). Le concept de groupe et la théorie de la perception. Journal de Psychologie, 368-414.

Cassirer, E. (1944). The Concept of Group and the Theory of Perception. Philosophy and Phenomenological Research 5(1), 1-36.

Cassirer, E. (1950). The Problem of Knowledge; Philosophy, Science, and History since Hegel. New Haven: Yale University Press.

Christoffel, E. B. (1869). Ueber die Transformation der homogenen Differentialausdrücke zweiten Grades. Journal für die reine und angewandte Mathematik 70, 46-70. now in Christoffel, 1910, vol I, 352-377, 378-382.

Christoffel, E. B. (1910). Gesammelte mathematische Abhandlungen, Volume L. Maurer. Leipzig: Teubner.

Coffa, A. J. (1979). Elective Affinities: Weyl and Reichenbach. In W. C. Salmon (Ed.), Hans Reichenbach: Logical Empirist. Dordrecht, Boston, London: Kluwer.

CPAE: Einstein, A. (1996-). The Collected Papers of Albert Einstein. Princeton: Princeton University Press.

D'Agostino, S. (2000). A History of the Ideas of Theoretical Physics : Essays on the Nineteenth and Twentieth Century Physics. Boston studies in the philosophy of science ; 213. Dordrecht: Kluwer Academic.

Darrigol, O. (2007). A Helmholtzian Approach to Space and Time. Studies In History and Philosophy of Science Part A 38(3), 528542 .

Earman, J. and C. Glymour (1980). The Gravitational Red Shift as a Test of General Relativity: History and Analysis. Studies In History and Philosophy of Science Part A 11(3), 175-214.

Ehrenfest, P. (1909). Gleichförmige Rotation starrer Körper und Relativitätstheorie. Physikalische Zeitschrift $10,918$. 
Einstein, A. (1905). Zur Elektrodynamik bewegter Körper. Annalen der Physik 17, 891-921. now in CPAE 2, Doc. 23.

Einstein, A. (1907). Relativitätsprinzip und die aus demselben gezogenen Folgerungen. Jahrbuch der Radioaktivität 4, 411-462. now in CPAE 2, Doc. 47.

Einstein, A. (1911). Die Relativitäts-Theorie. Naturforschende Gesellschaft, Zürich, Vierteljahresschrift $56,1-14$. now in CPAE 3, Doc. 17.

Einstein, A. (1912). Lichtgeschwindigkeit und Statik des Gravitationsfeldes The Speed of Light and the Statics of the Gravitational Field. Annalen der Physik 38, 355-369.

Einstein, A. (1914). Die formale Grundlage der allgemeinen Relativitätstheorie. Preussische Akademie der Wissenschaften, Sitzungsberichte -, 1030-1085. now in CPAE 6, Doc. 9.

Einstein, A. (1915a). Erklärung der Perihelbewegung des Merkur aus der allgemeinen Relativitätstheorie. Preussische Akademie der Wissenschaften, Sitzungsberichte -, 831-839. now in CPAE 6, Doc. 24

Einstein, A. (1915b). Feldgleichungen der Gravitation. Preussische Akademie der Wissenschaften, Sitzungsberichte -, 844-847. now in CPAE 6, Doc. 25.

Einstein, A. (1915c). Grundgedanken der allgemeinen Relativitätstheorie und Anwendung dieser Theorie in der Astronomie. Preussische Akademie der Wissenschaften, Sitzungsberichte -, 315

Einstein, A. (1915d). Zur allgemeinen Relativitätstheorie. Preussische Akademie der Wissenschaften, Sitzungsberichte -, 778-786, 799-801. now in CPAE 6, Doc. 21 and 22.

Einstein, A. (1916). Die Grundlage der allgemeinen Relativitätstheorie. Annalen der Physik 49, 769-822. now in CPAE 6, Doc 30 .

Einstein, A. (1918). Nachtrag zu H. Weyl, Gravitation und Elektrizität. Sitzungsberichte der Preussischen Akademie der Wissenschaften -, 478-80.

Einstein, A. (1921). Geometrie und Erfahrung. Erweiterte Fassung des Festvortrages gehalten an der Preussischen Akademie der Wissenschaften zu Berlin am 27. Januar 1921. Berlin: Springer. now in CPAE 7, Doc. 52.

Einstein, A. (1923). Grundgedanken und Probleme der Relativitätstheorie. Nobel prize lecture, delivered before the Nordische Naturforscherversammlung in Göteborg.

Einstein, A. (1924a). Über den Äther. Verhandlungen der Schweizerischen Naturforschenden Gesellschaft 105, 85-93.

Einstein, A. (1924b). Review of Elsbach, 1924. Deutsche Literaturzeitung 45, 1685-1692.

Einstein, A. (1925). Nichteuklidische Geometrie und Physik. Die neue Rundschau 36, 16-20.

Einstein, A. (1926). Space-time. In Encyclopedia Britannica (13 ed.)., pp. 608-609. Chicago: Encyclopædia Britannica, Inc.

Einstein, A. (1930a). Das Raum-, Feld- und Ather-Problem in der Physik. Gesamtbericht, Zweite Weltkraftkonferenz 19, 1-5.

Einstein, A. (1930b). Das Raum-, Feld- und Ather-Problem in der Physik. Koralle $\sim$ 5, 486-487.

Einstein, A. (1930c). Raum, Äther und Feld in der Physik. Forum Philosophicum 1, 173-180. tr. the same volume, pp. 180-184.

Einstein, A. (1934). Das Raum-, Äther- und Feld-Problem der Physik. In C. Seelig (Ed.), Mein Weltbild. Amsterdam: Querido Verlag.

Einstein, A. (1936). Physik und Realität/Physics and Reality. Franklin Institute, Journal 221, 313-347; English translation (by J Picard), 349-382. Also reprinted in Zeitschrift für freie deutsche Forschung, 1, no. 1, pp. 5-19 and no. 2, pp. 1-14 (1938).

Einstein, A. (1949a). Autobiographical notes. In P. A. Schilpp (Ed.) Albert Einstein, Philosopher-Scientist. Evanston, Ill.: Library of Living Philosophers.

Einstein, A. (1949b). Remarks Concerning the Essays Brought together in this Co-operative Volume. In P. A. Schilpp (Ed.), Albert Einstein, Philosopher-Scientist. Evanston, Ill.: Library of Living Philosophers.

Einstein, A. (1954). Ideas and Opinions. New York: Crown Publishers.
Einstein, A. and M. Grossmann (1913). Entwurf einer verallgemeinerten Relativitätstheorie und eine Theorie der Gravitation. I. Physikalischer Teil von A. Einstein II. Mathematischer Teil von M. Grossmann. Zeitschrift für Mathematik und Physik 62, 225244, 245-261. now in CPAE 4, 13.

Elsbach, A. C. (1924). Kant und Einstein: Untersuchungen über das Verhältnis der modernen Erkenntnistheorie zur Relativitätstheorie. Berlin: de Gruyter.

Flamm, L. (1916). Beiträge zur Einstein'schen Gravitationstheorie. Physikalische Zeitschrift $17,448-454$.

Fogel, B. (2008). Epistemology of a Theory of Everything. Weyl, Einstein, and the Unification of Physics. Notre Dame: University of Notre Dame, Diss.

Frank, P. (1949). Einstein's Philosophy of Science. Reviews of Modern Physics 21 (3), 349.

Freudenthal, H. (1956). Neuere Fassungen des Riemann-HelmholtzLieschen Raumproblems. Mathematische Zeitschrift 63, 374-405.

Freundlich, E. (1920). Zu dem Aufsatze „Die Physik als geometrische Notwendigkeit" von Arthur Haas (Naturwissenschaften 1920, Heft 3). Naturwissenschaften 8, 234-235. 10.1007/BF02448046.

Friedman, M. (1995). Poincaré's Conventionalism and the Logical Positivists. Foundations of Science 2, 299-314. now in [Friedman 1999].

Friedman, M. (2002). Geometry as a Branch of Physics. Background and Context for Eisnstein's 'Geometry and Experience'. In D. Malament (Ed.), Reading Natural Philosophy. Chicago-La Salle (Ill.): Open Court.

Giedymin, J. (1982). On the Origin and Significance of Poincaré's Conventionalism. In . ; (Ed.), Science and convention. Essay on Henri Poincaré's philosophy of science and the conventionalist tradition. Oxford: Pergamon Press.

Goenner, H. F. (2004). On the History of Unified Field Theories. Living Reviews in Relativity 7(2).

Grøn, Ø. (2004.). Space Geometry in a Rotating Reference Frame: A Historical Appraisal. In G. Rizzi and M. Ruggiero (Eds.), Relativity in Rotating Frames. Dordrecht: Kluwer.

Grünbaum, A. (1963a). Carnap's Views on the Foundations of Geometry. In P. A. H. Schilpp (Ed.), The philosophy of Rudolf Carnap, The Library of Living Philosophers ; 11. Lasalle, Ill. [et al.]: Open Court [et al.].

Grünbaum, A. (1963b). Philosophical Problems of Space and Time. Dordrecht, Boston, London: Reidel.

Grünbaum, A. (1968a). Geometry and chronometry: in philosophical perspective. Minneapolis: University of Minnesota Press.

Grünbaum, A. (1968b). Reply to Hilary Putnam's 'An Examination of Grnbaum's Philosophy of Geometry'. In R. Cohen and M. Wartofsky (Eds.), Boston Studies in the Philosophy of Science, Volume 5 , pp. 1-150. Reidel.

Hecht, H. and D. Hoffmann (1982). Die Berufung Hans Reichenbachs an die Berliner Universität. Deutsche Zeitschrift für Philosophie 30, 651-662.

Heinzmann, G. (2001). The Foundations of Geometry and the Concept of Motion: Helmholtz and Poincaré. Science in Context 14 (3), 457-470.

Helmholtz, H. (1921). Schriften zur Erkenntnistheorie. Berlin: Springer.

Hentschel, K. (1982). Zur Rolle Hans Reichenbachs in den Debatten um die Relativitätstheorie (mit der vollständigen Korrespondenz Reichenbach-Friedrich Adler im Anhang). Nachrichtenblatt der Deutschen Gesellschaft für Geschichte der Medizin, Naturwissenschaft \& Technik 3, 101-.

Hentschel, K. (1986). Die Korrespondenz Einstein-Schlick: Zum Verhältnis der Physik zur Philosophie. Annals of Science 43, 475488

Hentschel, K. (1994). Erwin Finlay Freundlich and Testing Einstein's Theory of Relativity. Archive for the History of the Exact Sciences 47, 143-201.

Herglotz, G. (1910). Über den vom Standpunkt des Relativitätsprinzips aus als "starr" zu bezeichnenden Körper. Annalen der Physik 31, 393-415.

Hilbert, D. (1916/1917, Wintersemester). Die Grundlagen der Physik 
II. Georg-August-Universitat Göttingen, Mathematisches Institut, Lesesaal (now in Hilbert 2009, 162-307).

Hilbert, D. (2009). David Hilbert's Lectures on the Foundations of Physics 1915-1927. Springer Berlin Heidelberg.

Howard, D. (1984). Realism and Conventionalism in Einstein's Philosophy of Science: The Einstein-Schlick Correspondence. Philosophia naturalis 21, 618-29.

Howard, D. (1990). Einstein and Duhem. Synthese 83, 363-384.

Howard, D. (1994). Einstein, Kant and the Origins of Logical Empiricism. In W. C. Salmon and G. Wolters (Eds.), Logic, Language, and the Structure of Scientific theories: Proceedings of the Carnap-Reichenbach Centennial, University of Konstanz, 21-24 May 1991. Pittsburgh, Pa.; Konstanz: University of Pittsburgh Press; Universitätsverlag Konstanz.

Howard, D. (2005). Einstein's Philosophy of Science. In E. N. Zalta (Ed.), The Stanford Encyclopedia of Philosophy (Spring 2004 Edition). - .

Howard, D. (2009). Einstein and the Development of TwentiethCentury Philosophy of Science. In M. Janssen and C. Lehner (Eds.), The Cambridge Companion to Einstein. Cambridge: Cambridge University Press.

Howard, D. (2010). "Let Me Briefly Indicate Why I Do Not Find This Standpoint Natural" : Einstein, General Relativity, and the Contingent a Priori. In Discourse on a New Method: Reinvigorating the Marriage of History and Philosophy of Science. Open Court.

Jackson, C. V. (1936). The Red Line of Cadmium as a Standard of Wave-Length. Proceedings of the Royal Society of London. Series A, Mathematical and Physical Sciences 155(885), 407-419.

Kaluza, T. (1910). Zur Relativitätstheorie. Physikalische Zeitschrift 11, 977-978.

Laue, M. $\sim$ v. (1920). Theoretisches über neuere optische Beobachtungen zur Relativitätstheorie. Physikalische Zeitschrift 21, 659662

Levi-Civita, T. and G. Ricci-Curbastro (1900). Méthodes de calcul différentiel absolu et leurs applications. Mathematische Annalen 54, 125-201. now in Ricci-Curbarstro 1956-57, I,.

Lie, S. (1886). Bemerkungen zu v. Helmholtz' Arbeit über die Tatsachen, welche der Geometrie zugrunde liegen. Berichte über die Verhandlungen der Kgl. Sächsischen Gesellschaft der Wissenschaften zu Leipzig 38, 337-342.

Lie, S. (1893). Theorie der Transformationsgruppen, Volume 3:. Leipzig: Teubner

Lipschitz, R. (1869). Untersuchungen in Betreff der ganzen homogenen Functionen von $\mathrm{n}$ Differentialen. Journal für die reine und angewandte Mathematik 70, 71-102.

London, F. (1927). Die Theorie von Weyl und die Quantenmechanik. Naturwissenschaften 15, 187-187.

Lorentz, H. A. (1917). On Einstein's Theory of Gravitation I. Proc. Amst. Soc. 19(II), 1341-1354.

Maltese, G. and L. Orlando (1995). The Definition of Rigidity in the Special Theory of Relativity and the Genesis of the General Theory of Relativity. Studies In History and Philosophy of Science Part B: Studies In History and Philosophy of Modern Physics 26(3), $263-306$.

Nagel, E. (1950). Einstein's Philosophy of Science. The Kenyon Review 12(3), 520-531.

Noether, F. (1910). Zur Kinematik des starren Körpers in der Relativitätstheorie. Annalen der Physik 31, 919-944,.

Norton, J. D. (1999). Geometries in Collision: Einstein, Klein and Riemann. In J. Gray (Ed.), The Symbolic Universe, pp. 128-144. Oxford; New York: Oxford University Press.

O'Raifeartaigh, L. and N. Straumann (2000). Gauge theory: Historical Origins and Some Modern Developments. Reviews of Modern Physics 72, 1-23.

Pais, A. (1982). Subtle is the Lord: The Science and the Life of Albert Einstein. New York: Oxford University Press.

Parrini, P. (2005). L'empirismo logico. Aspetti storici e prospettive teoriche. Roma: Carocci.

Pesic, P. (Ed.) (2007). Beyond geometry: Classic Papers from Riemann to Einstein. Mineola, N.Y.: Dover Publications.
Putnam, H. (1963). An Examination of Grünbaum's Philosophy of Space and Time. In B. Baumrin (Ed.), Philosophy of Science. The Delaware Seminar vol. 2, 1962-1963, pp. 205-255. New York: Interscience/John Wiley. now in Putnam, 1975, 93-129.

Reich, K. (1992). Levi-Civitasche Parallelverschiebung, affiner Zusammenhang, Übertragungsprinzip: 1916/17-1922/23. Archiv für Geschichte der Philosophie 44(1), 77-105.

Reich, K. (1994). Die Entwicklung des Tensorkalküls: vom absoluten Differentialkalkül zur Relativitätstheorie. Berlin: Birkhäuser.

Reichenbach, H. (1920). Relativitätstheorie und Erkenntnis apriori. Springer.

Reichenbach, H. (1921). Der gegenwärtige Stand der Relativitätsdiskussion. Eine kritische Untersuchung. Logos 22(10), 316-378.

Reichenbach, H. (1922). La signification philosophique de la théorie de la relativité. Revue philosophique de la France et de l'Étranger 93, 5-61.

Reichenbach, H. (1924). Axiomatik der relativistischen Raum-ZeitLehre. Vieweg: Braunschweig.

Reichenbach, H. (1928). Philosophie der Raum-Zeit-Lehre. Berlin and Leipzig: Walter de Gruyter. now in Reichenbach 1977, vol II.

Reichenbach, H. (1929a). Ziele und Wege der physikalischen Erkenntnis. In Handbuch der Physik, vol. 4: Allgemeine Grundlagen der Physik, pp. 1-80. Berlin: Springer.

Reichenbach, H. (1929b). Zur Einordnung des neuen Einsteinschen Ansatzes über Gravitation und Elektrizität. Zeitschrift für Physik 59(nos. 9-10), 683-689.

Reichenbach, H. (1949). The Philosophical Significance of the Theory of Relativity. In P. A. Schilpp (Ed.), Albert Einstein. PhilosopherScientist, pp. 289-311. New York: Tudor.

Reichenbach, H. (1951). The Rise of Scientific Philosophy. Berkely/Los Angeles/London: University of California Press.

Reichenbach, H. (1958). The Philosophy of Space and Time. New York: Dover Publ.

Reichenbach, H. (1969). Axiomatization of the Theory of Relativity. Berkeley,: University of California Press.

Reichenbach, H. (1977). Gesammelte Werke in 9 Bänden. Braunschweig; Wiesbaden: Vieweg.

Reichenbach, H. (1978). Selected Writings: 1909 - 1953. Vienna circle collection ; 4. Dordrecht: Reidel.

Ricci-Curbastro, G. (1883). Principii di una teoria delle forme differenziali quadratiche. Annali di Matematica Pura ed Applicata 12, 135-167. now in Ricci-Curbarstro 1956-57, I,.

Ricci-Curbastro, G. (1886). Sui parametri e gli invarianti delle forme quadratiche differenziali. Annali di Matematica Pura ed Applicata (1867 - 1897) 14, 1-11. now in Ricci-Curbarstro 1956-57, I,.

Ricci-Curbastro, G. (1888). Delle Derivazioni covarianti e controvarianti e del loro uso nella analisi applicata. In Studi editi dalla Università di Padova a commemorare l'ottavo centenario della Università di Bologna, Volume 3, pp. 3-23. Padova: Tip. del Seminario.

Ricci-Curbastro, G. (1889). Sopra certi sistemi di funzioni. Atti Accad. Lincei 4/6, 112-118. now in Ricci-Curbarstro 1956-57, I,

Ricci-Curbastro, G. (1892). Le calcul différentiel absolu. Bulletin des sciences mathématiques 16, 167-189.

Ricci-Curbastro, G. (1956-57). Opere. Roma: Cremonese.

Ryckman, T. (1995). Weyl, Reichenbach and the Epistemology of Geometry. Studies in History and Philosophy of Science 25(6), 831-870.

Ryckman, T. (1996). Einstein Agonists: Weyl and Reichenbach on Geometrv and the General Theory of Relativity. In R. N. Giere (Ed.), Origins of Logical Empiricism, pp. 165-209. Minneapolis: University of Minnesota Press.

Ryckman, T. (2005). The Reign of Relativity. Philosophy in Physics 1915-1925. Oxford, New York: Oxford University Press.

Rynasiewicz, R. (2005). Weyl vs. Reichenbach on Lichtgeometrie. In A. J. Kox and J. Eisenstaedt (Eds.), The Universe of General Relativity. Boston: Birkhäuser.

Sauer, T. (2006). Field Equations in Teleparallel Space-Time: Einstein's Fernparallelismus Approach toward Unified Field Theory. Historia Mathematica 33(4), 399 - 439.

Sauer, T. (2008). The Einstein-Varićak Correspondence on Rel- 
ativistic Rigid Rotation. In R. J. H. Kleinert and R. Ruffini (Eds.), The Eleventh Marcel Grossmann Meeting on recent Developments in Theoretical and Experimental General Relativity, Gravitation and Relativistic Field Theories. Singapore; Hackensack, N.J.: World Scientific Pub. Co.

Schlick, M. (1917). Raum und Zeit in der gegenwärtigen Physik. Zur Einführung in das Verständnis der allgemeinen Relativitätstheorie. Die Naturwissenschaften 5, 161-167; 177-186. now in Schlick, 2006, vol. II.

Schlick, M. (1918). Allgemeine Erkenntnisslehre. Naturwissenschaftliche Monographien und Lehrbücher. Berlin: J. Springer. now in Schlick 2006-, vol. I.

Schlick, M. (1921). Review of Einstein 1921. Die Naturwissenschaften 22, 435-436.

Schlick, M. (1925). Allgemeine Erkenntnisslehre (2 ed.). Naturwissenschaftliche Monographien und Lehrbücher. Berlin: J. Springer. now in Schlick 2006, vol. 1.

Schlick, M. (1978). Philosophical Papers. Vienna Circle collection ; 11. Dordrecht: Reidel.

Schlick, M. (2006-). Gesamtausgabe. Berlin: Springer.

Schlick, M. and H. Reichenbach (1920-22). Correspondence 192022.

Scholz, E. (2004). Hermann Weyl's Analysis of the "Problem of Space" and the Origin of Gauge Structures. Science in Context $\sim 17,165-197$.

Scholz, E. (2008). Weyl Geometry in Late 20th Century Physics. In D. E. Rowe (Ed.), Beyond Einstein. Proceedings Mainz Conference September 2008., pp. - Birkhäuser: Basel. to appear.

Schwarzschild, K. (1916). Über das Gravitationsfeld eines Massenpunktes nach der Einsteinschen Theorie. Sitzungsberichte der Königlich-Preussischen Akademie der Wissenschaften Sitzung vom 3. Februar 1916, 189-196.

Shapiro, L. S. (1994). 'Coordinative Definition' and Reichenbach's Semantic Framework: A Reassessment. Erkenntnis 41/3, 287323.

Stachel, J. (1989). The Rigidly Rotating Disk as the 'Missing Link in the History of General Relativity. In D. Howard and J. Stachel (Eds.), Einstein and the History of General Relativity (Einstein Studies, Vol. 1), pp. 48-62. Boston: Birkhäuser.

Torretti, R. (1978). Philosophy of Geometry from Riemann to Poincaré. Dordrecht; Boston; London: Reidel.

Torretti, R. (1983). Relativity and Geometry. Oxford: Pergamon Press.

Torretti, R. (1999). The Philosophy of Physics. Cambridge: Cambridge Univ. Press.

Vizgin, V. P. (1994). Unified Field Theories in the First Third of the 20th Century. Boston/Basel/Stuttgart: Birkhäuser.

von Laue, M. (1911). Zur Diskussion über den starren Körper in der Relativitätstheorie. Physikalische Zeitschrift 12, 85-87.

Weyl, H. (1918a). Erwiederung auf Einsteins Nachtrag zu H. Weyl, Gravitation und Elektrizität. Sitzungsberichte der Preussischen Akademie der Wissenschaften -, 478-80. now in WGA II, Doc. 31.

Weyl, H. (1918b). Gravitation und Elektrizität. Sitzungsberichte der preuß. Akad. Berlin, Math. Kl. -, 465-480. now in WGA II, Doc. 31.

Weyl, H. (1918c). Raum, Zeit, Materie: Vorlesungen über allgemeine Relativitätstheorie. Berlin: Springer.

Weyl, H. (1918s). Reine Infinitesimalgeometrie. Mathematische Zeitschrift 2, 384-411. now in WGA II, Doc. 30.

Weyl, H. (1919). Raum, Zeit, Materie: Vorlesungen über allgemeine Relativitätstheorie. Berlin: Springer. 2nd ed.

Weyl, H. (1920). Elektrizität und Gravitation. Physikalische Zeitschrift 21 (23/24), 649-650. now in WGA II, Doc. 40.

Weyl, H. (1921a). Über die physikalischen Grundlagen der erweiterten Relativitätstheorie. Physikalische Zeitschrift 22, 473-480. now in WGA II, Doc. 46.

Weyl, H. (1921b). Feld und Materie. Annalen der Physik 541-563, 65. now in WGA II, Doc. 47

Weyl, H. (1921c). Raum, Zeit, Materie: Vorlesungen über allgemeine Relativitätstheorie (4 ed.). Berlin: Springer.
Weyl, H. (1927). Philosophie der Mathematik und Naturwissenschaft. In A. Bäumler and M. SSchröter (Eds.), Handbuch der Philosophie, pp. - München/Berlin: Oldenbourg.

Weyl, H. (1929). Gravitation and the Electron. Proceedings of the National Academy of Sciences of the United States of America 15, 323-334

Weyl, H. (1949). Philosophy of Mathematics and Natural Science. Princeton: Princeton University Press.

WGA: Weyl, H. (1968). Gesammelte Abhandlungen. Berlin: Springer.

Yang, C.-N. (1986). Hermann Weyl's Contribution to Physics. In C. $\sim$ N. Yang, R. Penrose, A. Borel, and K. Chandrasekharan (Eds.), Hermann Weyl: 1885 - 1985; Centenary Lectures. Berlin: Springer. 